\title{
An ALMA/NOEMA study of gas dissipation and dust evolution in the 5 Myr-old HD 141569A hybrid disc *
}

\author{
E. Di Folco ${ }^{1,2}$, J. Péricaud ${ }^{1,2}$, A. Dutrey ${ }^{1,2}$, J.-C. Augereau ${ }^{3}$, E. Chapillon ${ }^{4}$, S. Guilloteau ${ }^{1,2}$, \\ V. Piétu ${ }^{4}$, and A. Boccaletti ${ }^{5}$ \\ ${ }^{1}$ Univ. Bordeaux, LAB, UMR 5804, 33270 Floirac, France \\ e-mail: emmanuel.di-folco@u-bordeaux.fr \\ ${ }^{2}$ CNRS, LAB, UMR 5804, 33270 Floirac, France \\ ${ }^{3}$ Univ. Grenoble Alpes, CNRS, IPAG, 38000 Grenoble, France \\ ${ }^{4}$ IRAM, 300 rue de la piscine, 38406 Saint Martin d'Hères, France \\ ${ }^{5}$ LESIA, Observatoire de Paris, PSL Research Univ., CNRS, Univ. Paris Diderot, Sorbonne Paris Cité, UPMC Paris 6 , \\ Sorbonne Univ., 5 place Jules Janssen, 92195 Meudon CEDEX, France
}

Received 6 November 2017 / Accepted 30 October 2019

\begin{abstract}
Context. The study of gas-rich debris discs is fundamental to characterising the transition between protoplanetary discs and debris discs.

Aims. We determine the physical parameters of the brightest gas-rich debris disc orbiting HD 141569A.

Methods. We analyse images from the NOrthern Extended Millimeter Array (NOEMA) ${ }^{1}$ and the Atacama Large Millimeter/ submillimeter Array (ALMA) in ${ }^{12} \mathrm{CO},{ }^{13} \mathrm{CO} \quad J=2 \rightarrow 1$, and ${ }^{13} \mathrm{CO} J=1 \rightarrow 0$ transitions. We incorporate ALMA archival data of the ${ }^{12} \mathrm{CO} J=3 \rightarrow 2$ transition and present continuum maps at $0.87,1.3$, and $2.8 \mathrm{~mm}$. We use simple parametric laws with the Diskfit code and MCMC exploration to characterise the gas disc parameters and report a first attempt to characterise its chemical content with IRAM-30 m.

Results. The continuum emission is equally shared between a compact ( $\leqslant 50 \mathrm{au}$ ) and a smooth, extended dust component ( $\sim 350 \mathrm{au}$ ). Large millimetre grains seem to dominate the inner regions, while the dust spectral index is marginally larger in the outer region. The ${ }^{12} \mathrm{CO}$ is optically thick, while ${ }^{13} \mathrm{CO}$ is optically thin with $\tau_{13 \mathrm{CO}} \sim 0.15\left(\mathrm{C}^{18} \mathrm{O}\right.$ is not detected $)$. The ${ }^{13} \mathrm{CO}$ surface density is constrained to be one order of magnitude smaller than around younger Herbig Ae stars, and we derive a gas mass $M_{1^{2} \mathrm{CO}}=10^{-1} M_{\oplus}$. We confirm the presence of a small $\mathrm{CO}$ cavity $\left(R_{\mathrm{CO}}=17 \pm 3 \mathrm{au}\right)$, and find a possibly larger radius for the optically thin ${ }^{13} \mathrm{CO} J=2 \rightarrow 1$ transition $(35 \pm 5 \mathrm{au})$. We show that the observed CO brightness asymmetry is coincident with the complex ring structures discovered with VLT/SPHERE in the inner $90 \mathrm{au}$. The ${ }^{12} \mathrm{CO}$ temperature $T_{0}(100 \mathrm{au}) \sim 30 \mathrm{~K}$ is lower than expected for a Herbig A0 star, and could be indicative of subthermal excitation.

Conclusions. With the largest amount of dust and gas among hybrid discs, HD 141569A shows coincident characteristics of both protoplanetary discs (central regions), and debris discs at large distance. Together with its morphological characteristics and young age, it appears to be a good candidate to witness the transient phase of gas dissipation, with an apparently large gas-to-dust ratio $(G / D>100)$ favouring a faster evolution of dust grains.
\end{abstract}

Key words. accretion, accretion disks - methods: observational - techniques: interferometric - protoplanetary disks stars: pre-main sequence - radio lines: stars

\section{Introduction}

The evolution of circumstellar discs from the initial reservoir of the interstellar medium (ISM)-dust and gas mixture towards planetary systems is governed by the growth and coagulation of dust grains, viscous spreading and accretion onto the central star (or on proto-planets), and dissipation of the primordial gas (Williams \& Cieza 2011). Although a large amount of high-resolution images and spectroscopic data have been collected during recent decades, the various stages of disc evolution remain poorly constrained by observations (Wyatt et al. 2015). The main accretion phase corresponds to the Class II phases for T Tauri (TTS) and Herbig Ae (HAe) stars. The final phase is represented by debris discs, when the primordial reservoir has been fully dissipated and/or incorporated into planetesimals

\footnotetext{
${ }^{\star}$ Reduced datacubes and images are only available at the CDS via anonymous ftp to cdsarc.u-strasbg.fr $(130.79 .128 .5)$ or via http://cdsarc.u-strasbg.fr/viz-bin/cat/J/A+A/635/A94
}

and planets, and the detected emission from second-generation dust comes from collisions between the leftovers of planet formation or comet evaporation (Wyatt 2008; Hughes et al. 2018). Evidence for structural changes has been collected for a large number of pre-main sequence stars at various stages of the disc evolution (e.g. Andrews et al. 2018; Avenhaus et al. 2018; Garufi et al. 2018). This includes the presence of spirals, rings and gaps, central cavities, or azimuthal asymmetries, where different processes are at play such as the protoplanet(s)-disc gravitational or hydrodynamical interaction, viscous evolution, grain growth and opacity change, gas-grain hydrodynamical interaction, the development of vortices and magneto-rotational instabilities, and the photo-evaporation of gas. Transitional discs with large dust cavities (e.g. Espaillat et al. 2014) were found to represent almost $30 \%$ of the millimetre(mm)-bright discs in Taurus and Ophiuchus regions (Andrews et al. 2011). These systems show a lack of continuum mm emission with dust depletion factors in the range of 100-1000 in the disc central regions, while a variety 
of gas depletion factors have been reported within these cavities (e.g. van der Marel et al. 2016, 2018). They may witness gravitational interaction between gas and sub-stellar/planetary companion(s) in the disc cavity, and drag forces between dust grains and gas may cause the mm grains to be trapped by pressure bumps at large radial distances (e.g. Zhu et al. 2011; Pinilla et al. 2012).

While the timescale for disc dissipation has been constrained to about $5 \mathrm{Myr}$ from the fading of infrared (IR)-excess (for dust) and of accretion tracers or molecular lines (for gas) in young clusters (Haisch et al. 2001; Fedele et al. 2010), very few systems are known in the transition phase between protoplanetary and debris discs. A new category of discs has emerged in recent years, which simultaneously display the typical characteristics of debris discs (with in particular a weak fractional excess of IR emission $f_{\mathrm{d}}=L_{\mathrm{IR}} / L_{\star}<10^{-2}$ originating from the optically thin dust component of second-generation grains), and the presence of gas tracers (mostly CO lines) that reveal a nonnegligible residual gaseous component (Zuckerman et al. 1995; Moór et al. 2011). Although only a handful of such gas-rich debris discs were known when we started the present project (namely HD 21997, 49 Ceti, $\beta$ Pictoris), this population has now grown to almost twenty members with typical ages in the range of 10-40 Myr (e.g. Lieman-Sifry et al. 2016; Moór et al. 2017). The origin of the remnant gas is still a subject of controversy: the age of the system must be compared to the lifetime of the detected molecular species, which itself depends on the UV/ $\mathrm{X}$-ray stellar/interstellar emission and on the (complex) shielding effects (Kral et al. 2017). Those systems harbouring supposedly primordial gas are called hybrid discs (Kóspál et al. 2013). The gas-rich debris discs revealed by CO lines simultaneously display a weak dust mm continuum and an IR excess characteristic of more evolved, optically thin debris discs. These systems are thought to witness a short-lived transient phase at the interface between proto-planetary and debris discs, when the material dissipates through photo-evaporation and accretion. In Péricaud et al. (2017), we reported a correlation between the CO flux density and the mm continuum emission for a broad variety of systems including T Tauri, Herbig Ae, transition discs, and even the proto-typical young debris disc $\beta$ Pic. The position of hybrid discs in the diagram, systematically above the correlation line, suggests a faster evolution of the dust in these systems, leading to a transient and unusual increase of the gas-to-dust ratio.

In this population, the Herbig B9.5V/A0Ve star HD 141569A holds a very peculiar and interesting position. The star is located at $110.6 \pm 0.5 \mathrm{pc}$ (Gaia Collaboration 2018), and has two M-stars companions whose gravitational link remains unclear (Reche et al. 2009). With an age of about $5 \pm 3 \mathrm{Myr}$ (Weinberger et al. 2000; Merín et al. 2004), it is the youngest debris disc in this population with an IR brightness twice as large as that of $\beta$ Pic. With the new Gaia distance, the stellar luminosity determined in Merín et al. (2004) turns to $27.0 \pm 3.6 L_{\odot}$. In IR colour-magnitude diagrams, HD 141569A lies at the exact interface between proto-planetary and debris discs (see, e.g. Wyatt et al. 2015, and Fig. 1 therein). Its cold molecular content was discovered through its $\mathrm{CO}$ emission line in the spectroscopic survey of Zuckerman et al. (1995), whereas warmer CO gas was later detected in the inner system by Goto et al. (2006). The debris disc of HD 141569A $\left(L_{\mathrm{IR}} / L_{\star}=8.4 \times 10^{-3}\right.$, Sylvester et al. 1996) revealed by optical/IR dust emission presents a complex multiple-ring architecture with spirals, arcs, and gaps imaged in scattered light by the HST (Augereau et al. 1999; Weinberger et al. 1999; Mouillet et al. 2001; Boccaletti et al. 2003; Clampin et al. 2003; Konishi et al. 2016). More recently, a series of concentric rings was discovered with VLT/SPHERE with a range of radii of 40-90 au (Perrot et al. 2016), partially corroborating the emission detected around 40 au in the $L^{\prime}$ band by Currie et al. (2016) with Keck/NIRC2.

While the small-grain component has been well studied in the near-infrared (NIR), a thorough analysis of the larger grains observed at $\mathrm{mm}$ wavelengths and a comparative physical and morphological analysis of the $\mathrm{CO}$ gas distribution is crucial to understanding its evolution status as well as the nature of the observed dust and gas components. Our group obtained observations of HD 141569A in 1999-2000 with the Plateau de Bure Interferometer (PdBI) of Institut de Radio-Astronomie Millimétrique (IRAM), where the gas disc was already partly resolved (briefly reported in Dutrey et al. 2004). These early observations have motivated the present study, but their quality and sensitivity is outmatched by the current dataset and will therefore not be presented here. Observations of this system by the Submilliter Array (SMA), Combined Array for Research in Millimeter-wave Astronomy (CARMA), and the Atacama Large Millimeter/submillimeter Array (ALMA) have been reported, revealing an extended gas disc (from the ${ }^{12} \mathrm{CO} J=3 \rightarrow 2$ transition) out to the 250 au main dust ring highlighted in early HST images, and a dust disc extending out to $56 \mathrm{AU}$ from the $870 \mu \mathrm{m}$ continuum observations (White et al. 2016; Flaherty et al. 2016). The total mass of gas was revised to values much larger than initially derived thanks to the optically thin line ${ }^{13} \mathrm{CO} J=2 \rightarrow 1$ (Miley et al. 2018). From the mm continuum emission, a double component with spectral indices consistent with the range of values observed in both debris and primordial discs was reported by White $\&$ Boley (2018), and an unresolved dust ring peaking at 220 au was detected from $1.3 \mathrm{~mm}$ ALMA observations by Miley et al. (2018). In this article, we present complementary, multifrequency observations with ALMA and the NOrthern Extended Millimeter Array (NOEMA) ${ }^{1}$ (Sect. 2). We characterise the dust $\mathrm{mm}$ continuum and the gas emission, taking advantage of the spectral information to derive constraints on the grain growth and emission lines opacities (Sect. 3). We finally model the ${ }^{12} \mathrm{CO}$ and ${ }^{13} \mathrm{CO}$ lines with the radiative transfer code Diskfit (Piétu et al. 2007) to characterise the temperature and density laws in this disc (Sect. 4). In Sect. 5, we finally compare the derived morphology at $\mathrm{mm}$ wavelengths with the dust disc seen in optical/IR domain and use the new constraints on the gas and dust mass to discuss the origin of the gas and the evolution status of this peculiar system.

\section{Observations}

\subsection{PdBI/NOEMA observations}

HD 141569 was observed with NOEMA during three runs from December 2014 to April 2016. The ${ }^{12} \mathrm{CO} J=2 \rightarrow 1$ transition at $230.538 \mathrm{GHz}$ was mapped with the 7D and 7C configurations (7 antennas) of the IRAM array, with an angular resolution of $2.4^{\prime \prime} \times 1.2^{\prime \prime}$ (baseline lengths from 15 to $192 \mathrm{~m}$; all subsequent angular resolution values correspond to natural weighting). The spectral setup used a narrow band $(20 \mathrm{MHz}$ bandwidth, resolution $0.20 \mathrm{~km} \mathrm{~s}^{-1}$ ). Excellent conditions are noteworthy for one track, with precipitable water vapour inferior to $1.5 \mathrm{~mm}$, and a $15^{\circ}$ mean phase rms.

In winter 2014/2015, we also obtained ${ }^{13} \mathrm{CO} \quad J=2 \rightarrow 1$ $(220.399 \mathrm{GHz})$ observations. The $6 \mathrm{C}$ and $7 \mathrm{D}$ configuration

1 Based on observations carried out with the IRAM Plateau de Bure Interferometer. IRAM is supported by INSU/CNRS (France), MPG (Germany), and IGN (Spain). 
antennas were used with a total on-source time of $12.7 \mathrm{~h}$. We reached a $2.3^{\prime \prime} \times 1.2^{\prime \prime}$ angular resolution (baseline lengths from 15 to $176 \mathrm{~m}$ ) and used the same spectral resolution as for ${ }^{12} \mathrm{CO} J=2 \rightarrow 1$. For both transitions, $1546+027$ and 1508-055 were used for amplitude and phase calibration and we bootstrapped their fluxes using MWC 349 observations (with a flux model of $1.86 \mathrm{Jy}$ at $219 \mathrm{GHz}$ and $1.91 \mathrm{Jy}$ at $230.5 \mathrm{GHz}$ ).

The WIDEX correlator provided $4 \mathrm{GHz}$ bandwidth during the observations of the ${ }^{12} \mathrm{CO}$ and ${ }^{13} \mathrm{CO}$. Combining the two observations at $1.3 \mathrm{~mm}$, we obtained a $2.1^{\prime \prime} \times 1.0^{\prime \prime}$ beam for the continuum map.

\subsection{ALMA observations}

We obtained ALMA observations of HD 141569 in Band 3 (84-116 GHz). The data were recorded in August 2015 for the ${ }^{13} \mathrm{CO} J=1 \rightarrow 0(110.201 \mathrm{GHz})$ and $\mathrm{C}^{18} \mathrm{O} J=1 \rightarrow 0(115.271 \mathrm{GHz})$ transitions during two runs with 39 and 44 antennas, respectively (project 2013.1.00883, PI. Péricaud). The spatial resolution was $0.76^{\prime \prime} \times 0.56^{\prime \prime}$ (baselines up to $1.55 \mathrm{~km}$ ), and the spectral resolution $0.2 \mathrm{~km} \mathrm{~s}^{-1}$. The data were collected with relatively poor weather conditions $(\mathrm{PWV} \sim 4 \mathrm{~mm})$. We used Ceres and $1550+054$ for the flux and amplitude calibration, 1550+0527 and $1517-2422$ for bandpass calibration and $1550+0527$ for the phase calibration.

In addition, we collected archival raw data from the project 2012.1.00698.S (PI. Boley) to complement our analysis in Band $7(275-373 \mathrm{GHz})$, which includes the ${ }^{12} \mathrm{CO} J=3 \rightarrow 2$ line at $345.796 \mathrm{GHz}$. A preliminary analysis of this data set was reported in White et al. (2016). Only a subset of the observations could be properly calibrated. The observations carried out with 32 antennas provide a resolution of $0.43^{\prime \prime} \times 0.35^{\prime \prime}$ (baselines up to $650 \mathrm{~m}$ ), and a spectral resolution of $0.8 \mathrm{~km} \mathrm{~s}^{-1}$. The flux and amplitude calibrator is Titan, the phase and bandpass calibrator is $1550+0527$. We used the CASA package to calibrate the ALMA data, and we carried out the imaging and analysis of all NOEMA and ALMA observations with the GILDAS software. The imaging was performed with natural weighting for both ALMA and NOEMA.

\subsection{A deep search for extra molecular lines with IRAM-30 m/EMIR}

We also used the EMIR detector on the IRAM $30 \mathrm{~m}$ antenna to investigate the molecular content of this hybrid disc (Project 049-15). The observations were performed in Summer 2015 with a total time of $20 \mathrm{~h}$ on source. Two frequency bands were used in this project: $87.720-93.500 \mathrm{GHz}$ and 140.520 $148.300 \mathrm{GHz}$. We used Wobbler switching, resulting in very flat baselines over the observed bandwidths. The frequency coverage included lines of $\mathrm{HCN} J=1-0, \mathrm{HCO}^{+} J=1-0$, and CS $J=3-2$ that are usually bright in discs around classical T Tauri (of order $0.5-1.0 \mathrm{Jy} \mathrm{km} \mathrm{s}^{-1}$, see Dutrey et al. 1997) but slightly fainter in Herbig Ae stars (e.g. MWC 480 and AB Aur, Piétu et al. 2007; Schreyer et al. 2008). No emission was detected. Assuming a similar line width to that of the $\mathrm{CO}$ lines $\left(7 \mathrm{~km} \mathrm{~s}^{-1} F W H M\right)$, the $3 \sigma$ detection threshold on the integrated line flux is $45 \mathrm{mJy} \mathrm{km} . \mathrm{s}^{-1}$ around $90 \mathrm{GHz}$, and $60 \mathrm{mJy} \mathrm{km} . \mathrm{s}^{-1}$ around $145 \mathrm{GHz}$.

\section{Results}

\subsection{Continuum emission}

The continuum emission is resolved at the three frequencies, although with some differences due to the different instrumental
Table 1. Continuum results: best-fit parameters for: (i) a single component model (elliptical Gaussian in the $u, v$ plane), (ii) and a doublecomponent model (two circular Gaussian functions after deprojection in the $u, v$ plane according to the orientation and inclination determined at optical wavelength)

\begin{tabular}{|c|c|c|c|}
\hline & $0.87 \mathrm{~mm}$ & $1.3 \mathrm{~mm}$ & $2.8 \mathrm{~mm}$ \\
\hline \multicolumn{4}{|l|}{ Synthesized beam } \\
\hline Beam size $F W H M\left({ }^{\prime \prime}\right)$ & $0.43 \times 0.35$ & $2.1 \times 1.0$ & $0.76 \times 0.56$ \\
\hline Beam size $F W H M(\mathrm{au})$ & $47 \times 39$ & $231 \times 110$ & $84 \times 62$ \\
\hline Beam PA $\left(^{\circ}\right)$ & 121 & 14 & 57 \\
\hline \multicolumn{4}{|l|}{ Zero-baseline flux } \\
\hline$F_{v}(u, v \simeq 0, \mathrm{mJy})$ & \multirow[t]{2}{*}{$0.00 \pm 0.25$} & $4.00 \pm 0.25$ & \multirow[t]{2}{*}{$0.70 \pm 0.05$} \\
\hline Spectral index ${ }^{(*)}(\beta)$ & & $-0.16 \pm 0.29$ & \\
\hline Single-component fit & \multicolumn{3}{|c|}{ EGAUSS } \\
\hline$F_{v}$ (best-fit, mJy) & $3.93 \pm 0.15$ & $3.76 \pm 0.29$ & $0.39 \pm 0.02$ \\
\hline Semi-major axis $F W H M(\mathrm{au})$ & $54 \pm 2$ & $253 \pm 26$ & $35 \pm 4$ \\
\hline Semi-minor axis $F W H M(\mathrm{au})$ & $41 \pm 2$ & $156 \pm 13$ & $32 \pm 4$ \\
\hline Inclination $\left(^{\circ}\right)$ & $41 \pm 5$ & $53 \pm 6$ & 24 \\
\hline $\mathrm{PA}\left({ }^{\circ}\right)$ & $2 \pm 7$ & $21 \pm 8$ & $20 \pm 50$ \\
\hline Double-c & \multicolumn{3}{|c|}{ CGAUSS } \\
\hline$F_{v}^{\text {tot }}(1$ & $7.42 \pm 1.42$ & $4.20 \pm 1.24$ & $0.71 \pm 0.09$ \\
\hline$F_{v}^{\text {int }}($ best-fit, $\mathrm{mJy})$ & $3.02 \pm 0.16$ & $1.66 \pm 0.57$ & $0.32 \pm 0.02$ \\
\hline$F W H M^{\text {int }}(\mathrm{au})$ & $35 \pm 2$ & $110 \pm 33$ & $28 \pm 6$ \\
\hline Spectral index ${ }^{(*)}\left(\beta^{\text {int }}\right)$ & \multicolumn{3}{|c|}{$-0.05 \pm 0.07$} \\
\hline$F_{v}^{\text {out }}$ (best-fit, mJy) & $4.4 \pm 1.3$ & $2.54 \pm 0.67$ & $0.39 \pm 0.07$ \\
\hline$F W H M^{\text {out }}(\mathrm{au})$ & $322 \pm 55$ & $418 \pm 121$ & $407 \pm 66$ \\
\hline $\begin{array}{l}\text { Spectral index }{ }^{(*)}\left(\beta^{\text {out }}\right) \\
\left(\text { fixed PA }=356^{\circ}, i=53^{\circ} \text { ) }\right.\end{array}$ & \multicolumn{3}{|c|}{$0.17 \pm 0.23$} \\
\hline
\end{tabular}

Notes. $F_{v}(u, v \simeq 0)$ corresponds to the maximum flux density measured at the shortest baseline for each frequency. ${ }^{(*)}$ To compute the spectral index, we add quadratically an arbitrary uncertainty of $10 \%$ of the flux values to account for the instrumental calibration.

resolutions. The images presented in Fig. 1 were produced using the CLEAN deconvolution algorithm with natural weighting. The emission appears compact, and centrally peaked, with a possible secondary component which looks more extended in the NOEMA map only (a low level plateau in the $1.3 \mathrm{~mm}$ image). The coarser resolution of the NOEMA map $(231 \times 110 \mathrm{au})$ is not the only reason: the visibility profiles presented in Fig. 1 clearly show a steep increase at the shortest baselines. This visibility rise at $u, v$ distance shorter than $50-100 \mathrm{~m}$ (present at the three frequencies) is characteristic of extended emission (at few arcsec scales), while the more compact, inner disc component is resolved at intermediate baselines (e.g. $B \sim 150-400 \mathrm{~m}$ at $0.87 \mathrm{~mm}$ ). The spatial extent and flux values of the continuum emission were determined by fitting Gaussian functions in the $u, v$ plane. We tested a single and a two-component model. In Table 1 we present the best-fit parameters for the two models, and the measured maximum flux density corresponding to the shortest baseline points (so called zero-baseline flux). The single-component model is dominated by the compact disc component $\left(0.3-0.5^{\prime \prime}\right)$ at 0.87 and $2.8 \mathrm{~mm}$, while at $1.3 \mathrm{~mm}$ only the broad component $\left(2-3^{\prime \prime}\right)$ is constrained. Therefore, the flux values for this model cannot be used together to estimate the dust spectral index. Our maximum flux values from the shortest baselines are consistent with those reported by single-dish observations or compact arrays (see Table 2). At $1.3 \mathrm{~mm}$, we measure $F_{230 \mathrm{GHz}}(u, v \simeq 0)=4.00 \pm 0.25 \mathrm{mJy}$, in agreement with the JCMT/SCUBA measurement $(5.4 \pm 1.0 \mathrm{mJy}$, 

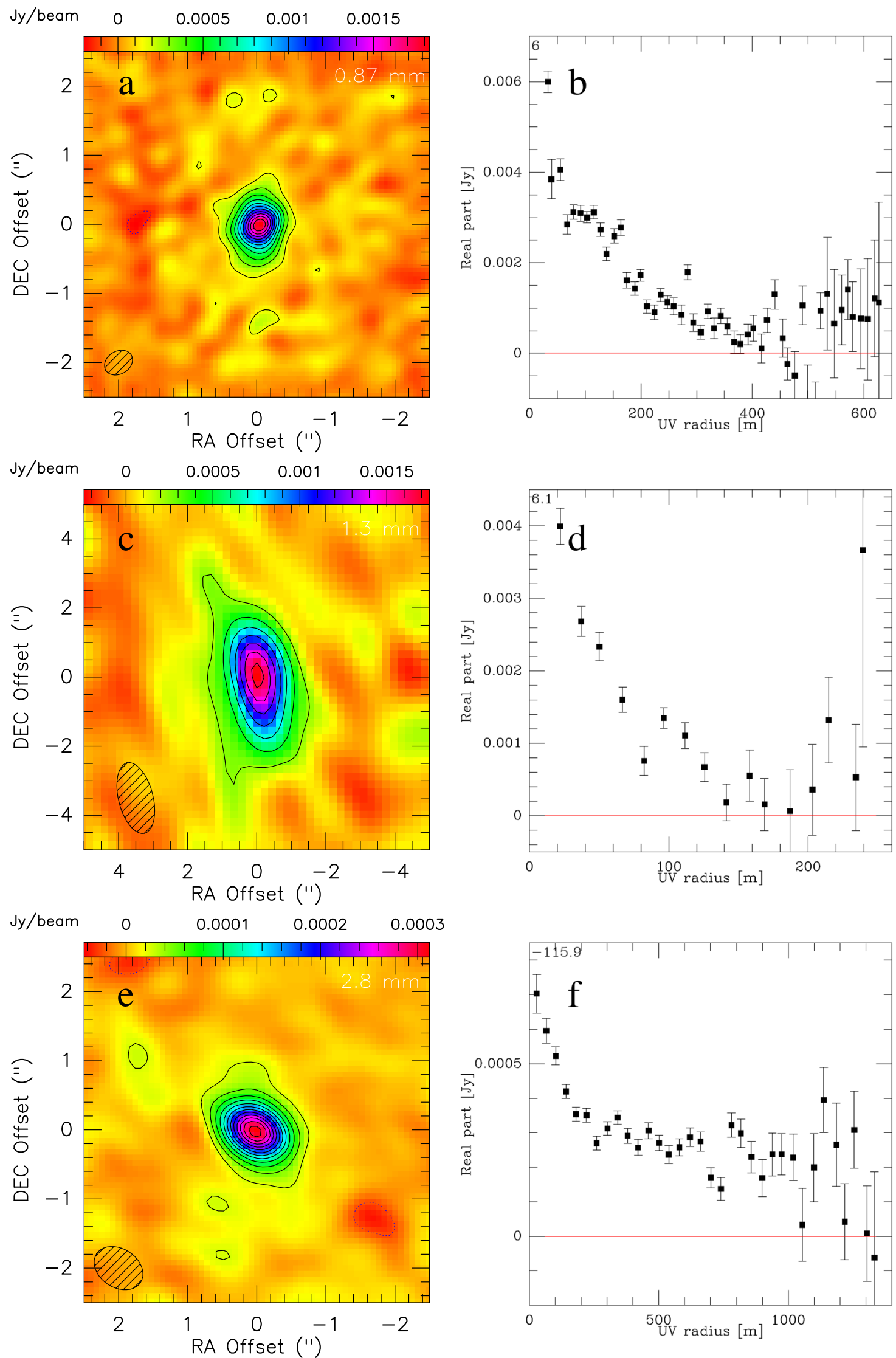

Fig. 1. Left column: continuum emission maps, all contours are set with $3 \sigma$ spacing, panel a: ALMA data at $0.87 \mathrm{~mm}^{-} \sigma=0.051 \mathrm{mJy}$ beam ${ }^{-1}$, contours; (c) NOEMA data at $1.3 \mathrm{~mm}, \sigma=0.078 \mathrm{mJy} \mathrm{beam}^{-1}$; (e) ALMA data at $2.8 \mathrm{~mm}, \sigma=0.008 \mathrm{mJy} \mathrm{beam}^{-1}$. We note that the scale of panel $c$ is changed to include the more extended emission. Right column: real part of the visibility (with spatial binning) as a function of the $u, v$ radius in the Fourier plane, panels $b, d$, and $f$ : correspond to data in panels a, c, and e, respectively. 
Table 2. Continuum flux comparison.

\begin{tabular}{|c|c|c|c|c|c|}
\hline Instrument & Beam $\left({ }^{\prime \prime}\right)$ & $F_{v}^{\text {tot }}(\mathrm{mJy})$ & $F_{v}^{\text {int }}(\mathrm{mJy})$ & $F_{v}^{\text {out }}(\mathrm{mJy})$ & Ref \\
\hline \multicolumn{6}{|l|}{$\lambda=0.87 \mu \mathrm{m}$} \\
\hline APEX & 19.2 & $12.6 \pm 4.6$ & & & 1 \\
\hline SMA & $1.7 \times 1.2$ & $8.2 \pm 2.4$ & & & 2 \\
\hline ALMA & $0.4 \times 0.3$ & $3.8 \pm 0.4$ & & & 3 \\
\hline ALMA & $0.4 \times 0.3$ & $16.8 \pm 1.7$ & $5.8 \pm 0.5$ & $11.0 \pm 1.2$ & 4 \\
\hline ALMA & $0.4 \times 0.3$ & $7.4 \pm 1.4$ & $3.0 \pm 0.2$ & $4.4 \pm 1.3$ & - \\
\hline \multicolumn{6}{|l|}{$\lambda=1.3 \mu \mathrm{m}$} \\
\hline JCMT & 22.0 & $5.4 \pm 1.0$ & & & 5 \\
\hline ALMA & 0.65 & 1.7 & 1.7 & & 6 \\
\hline ALMA & $1.0 \times 1.0$ & 7.0 & 2.8 & 4.2 & 4 \\
\hline NOEMA & $2.1 \times 1.0$ & $4.2 \pm 1.2$ & $1.7 \pm 0.6$ & $2.5 \pm 0.7$ & - \\
\hline \multicolumn{6}{|l|}{$\lambda=2.8 \mu \mathrm{m}$} \\
\hline CARMA & $5.1 \times 4.2$ & $0.8 \pm 0.3$ & & & 2 \\
\hline ALMA & $0.7 \times 0.5$ & $0.42 \pm 0.05$ & & & 7 \\
\hline ALMA & $0.7 \times 0.5$ & 1.25 & 0.62 & 0.63 & 4 \\
\hline ALMA & $0.8 \times 0.6$ & $0.7 \pm 0.1$ & $0.32 \pm 0.02$ & $0.39 \pm 0.07$ & - \\
\hline
\end{tabular}

References. (1) Nilsson et al. (2010); (2) Flaherty et al. (2016); (3) White et al. (2016); (4) White \& Boley (2018); (5) Sylvester et al. (2001); (6) Miley et al. (2018); (7) White et al. (2018); (-) this work: best-fit values from the two-component model.

Sylvester et al. 2001), but our value is about three times larger than the ALMA measurement of $1.7 \mathrm{mJy}$ reported in Miley et al. (2018). Typical calibration uncertainties would amount to $\sim 10 \%$ for both instruments, but cannot justify this discrepancy. At $2.8 \mathrm{~mm}$ (and $0.87 \mathrm{~mm}$ resp.) our measurements are consistent with the CARMA (resp. SMA) values $F_{110 \mathrm{GHz}}=0.78 \pm$ $0.30 \mathrm{mJy}$ (resp. $F_{230 \mathrm{GHz}}=8.2 \pm 2.4 \mathrm{mJy}$, Flaherty et al. 2016). Because the inner component is very compact, the disc orientation and aspect ratio are only roughly reproduced by the elliptical Gaussian fit in the single component model (see Table 1). In the more realistic double component model below, we therefore first deprojected the visibilities assuming the geometric parameters derived at optical wavelength $\left(\mathrm{PA}=356^{\circ}, i=53^{\circ}\right.$, Augereau et al. 1999), and then fit in the $u, v$ plane with a circular Gaussian function.

The second model suggests that the emission is about equally shared between a compact component, which is concentrated within about 50 au $\left(F W H M^{\text {int }} \sim 30-35\right.$ au, a value comparable to the interferometric beams at 0.87 and $2.8 \mathrm{~mm}$ ), and a much broader component that extends out to about $350 \mathrm{au}$. The $u, v$ plane coverage does not allow precise determination of the outer radius, and most of the flux of this extended component is filtered by the interferometer at 0.87 and $2.8 \mathrm{~mm}$. It is marginally detected as a low-level plateau in the $1.3 \mathrm{~mm}$ map thanks to the larger beam (although its shape and radial extent cannot be fully determined in our data). From higher-resolution ALMA data $\left(0.65^{\prime \prime}\right.$ beam) at $1.3 \mathrm{~mm}$, Miley et al. (2018) revealed a lowlevel ring-like emission peaking at $220 \pm 10$ au that cannot be seen in our NOEMA map. This unresolved ring is different from the extended component that we report from our analysis of the $u, v$ plane. In our higher-resolution maps at 0.87 and $2.8 \mathrm{~mm}$, no such ring-like emission is detected. Assuming a spectral index $\alpha_{\mathrm{mm}}=2$ (see below), and extrapolating the 220 au ring peak brightness and our brightness sensitivity at $0.87 \mathrm{~mm}\left(0.4^{\prime \prime}\right.$ beam $)$ and $2.8 \mathrm{~mm}\left(0.66^{\prime \prime}\right.$ beam $)$, it would be marginally detectable at the $\sim 3$ and $5 \sigma$ level, respectively. Such a two-component model was also proposed by White \& Boley (2018) from $u, v$ plane fitting, but our results disagree with their total flux values (e.g. $F_{345 \mathrm{GHz}}=16.8 \pm 1.7 \mathrm{mJy}$ ), which are systematically larger than both the zero-baseline flux values, and the earlier SMA, APEX, CARMA or JCMT observations (Flaherty et al. 2016; Nilsson et al. 2010; Sylvester et al. 2001). The observed differences between the flux values summarized in Table 2 can be explained firstly by the assumed shape of the model components (Gaussian, uniform discs, rings, power-laws), and the number of emission components taken into account. Secondly, the veryshort-baseline information is not always taken into account in the data analysis (e.g. White et al. 2016; Miley et al. 2018). Thirdly, the $u, v$ coverage and the field of view can vary significantly between instruments, and the signal-to-noise ratio as a function of $(u, v)$ radius can be very different from one dataset to another (e.g. at $1.3 \mathrm{~mm}$ ).

We estimate the dust spectral index $\beta$ from the slope of the spectral flux density, and we first add quadratically a typical calibration uncertainty of $10 \%$ to the flux density values. The spectral index is defined as $\beta=\alpha-2$, with $\alpha=$ $\log \left(F_{v_{1}} / F_{v_{2}}\right) / \log \left(v_{1} / v_{2}\right), F_{v}$. A fit to the three frequencies yields the slope $\alpha$. We derive the spectral index $\beta$ in two cases: (i) for the global disc using the maximum flux values measured at the shortest baselines $(u, v) \sim 0$, (ii) for the double component model, which provides a more realistic description of the continuum emission than the single-component model. The values reported in Table 1 suggest that the spectral index is significantly smaller than the canonical ISM value with $\beta \leq 0.7$ at the $3 \sigma$ level. Whether this is indicative of grain growth or optically thick emission can only be determined with more resolved observations. In the disc modelling by Thi et al. (2014), the dust component is optically thin from optical up to $\mathrm{mm}$ wavelength both in the radial and vertical directions, and we therefore expect the constraints on the spectral index to primarily reflect a change in grain properties. Radial variations of this spectral index may also be expected, as already demonstrated in a sample of TTS and HAe stars (Guilloteau et al. 2011; Pérez et al. 2012). The double-component model suggests a larger value of $\beta$ for the more extended disc component, although the difference with the inner 35 au region is not statistically significant. A similar trend has been claimed by White \& Boley (2018), although their spectral index value for the outer component $\beta=0.28 \pm$ 0.18 may be biased by an overly large flux value at $345 \mathrm{GHz}$, as explained above. A dense $u, v$ coverage at both short and very long baselines would be required to firmly establish a change of dust properties between the inner compact and the extended dust disc components.

\subsection{CO gas emission}

The ${ }^{12} \mathrm{CO}$ and ${ }^{13} \mathrm{CO} J=2 \rightarrow 1$ transitions are detected with NOEMA at a high signal-to-noise ratio $(S / N \gtrsim 150$, and $S / N \gtrsim$ 20 respectively), whereas the ${ }^{13} \mathrm{CO} J=1 \rightarrow 0$ detection with ALMA is more marginally significant $(S / N \sim 5)$. Integrated intensities for all $\mathrm{CO}$ transitions are presented in Table 3 with their associated spatial resolution. The intensity we derive for the ALMA ${ }^{12} \mathrm{CO} J=3 \rightarrow 2$ observations $18.4 \pm 0.3 \mathrm{Jy} \mathrm{km} \mathrm{s}^{-1}$ is slightly larger than (but consistent within $2 \sigma$ with) the value reported by White et al. (2016) and by Flaherty et al. (2016) with the SMA. The intensity maps (zeroth moment) are displayed in Fig. 2 for the three transitions (natural weighting). The ${ }^{12} \mathrm{CO}$ emission appears slightly more extended in the NOEMA map because of the convolution by a larger beam. The ${ }^{13} \mathrm{CO} J=2 \rightarrow 1$ emission appears to be more compact than 
Table 3. Summary of CO lines detections and setups.

\begin{tabular}{lcccccc}
\hline \hline Line & $\begin{array}{c}\text { Freq } \\
(\mathrm{GHz})\end{array}$ & Array & $\begin{array}{c}\text { Beam size } \\
\left({ }^{\prime \prime}\right)\end{array}$ & $\begin{array}{c}\text { Beam PA } \\
\left({ }^{\circ}\right)\end{array}$ & $\begin{array}{c}\text { CO integrated intensity } \\
\left(\mathrm{Jy} \mathrm{km} \mathrm{s}^{-1}\right)\end{array}$ & $\begin{array}{c}\text { Noise level } \\
\left(\mathrm{mJy} \mathrm{beam}^{-1} \mathrm{~km} \mathrm{~s}^{-1}\right)\end{array}$ \\
\hline${ }^{13} \mathrm{CO} J=1 \rightarrow 0$ & 110.201 & ALMA & $0.70 \times 0.51$ & 58 & $0.16 \pm 0.03$ & 6.8 \\
$\mathrm{C}^{18} \mathrm{O} J=1 \rightarrow 0$ & 109.782 & ALMA & $0.70 \times 0.51$ & 58 & $<0.05$ & \\
${ }^{12} \mathrm{CO} J=1 \rightarrow 0$ & 115.271 & CARMA $^{(b)}$ & $4.8 \times 4.1$ & 29 & $1.6 \pm 0.2^{(b)}$ & 23 \\
\hline${ }^{13} \mathrm{CO} J=2 \rightarrow 1$ & 220.399 & NOEMA & $2.30 \times 1.20$ & -172 & $1.2 \pm 0.2$ & 31 \\
$\mathrm{C}^{18} \mathrm{O} J=2 \rightarrow 1$ & 219.560 & NOEMA & $2.30 \times 1.20$ & -172 & $<0.10$ & 40 \\
${ }^{12} \mathrm{CO} J=2 \rightarrow 1$ & 230.538 & NOEMA & $2.40 \times 1.20$ & 11 & $10 \pm 1$ & \\
${ }^{12} \mathrm{CO} J=3 \rightarrow 2$ & 345.796 & ALMA $^{(a)}$ & $0.43 \times 0.33$ & -58 & $18.4 \pm 0.3{ }^{(a)}$ & \\
\hline
\end{tabular}

Notes. ${ }^{(a)}$ Archival data from project ALMA.1.00698.S. For non detections, we provide $3 \sigma$ upper limits.

References. ${ }^{(b)}$ From Flaherty et al. (2016).
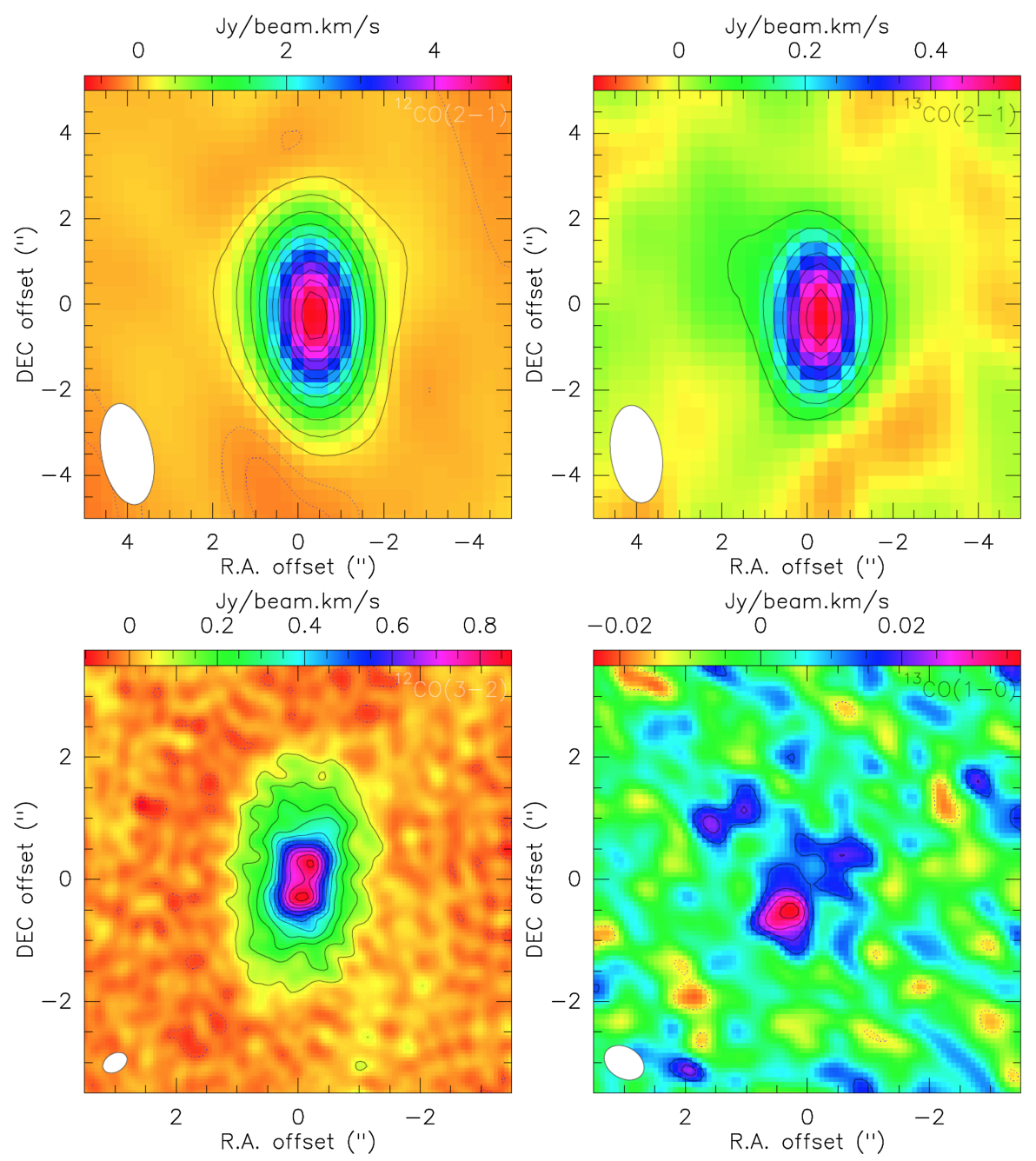

Fig. 2. Maps of integrated $\mathrm{CO}$ emission for the four detected transitions (the continuum was not subtracted due to its very low level of emission). Top: NOEMA observations, from left to right: ${ }^{12} \mathrm{CO} J=2 \rightarrow 1$ (first contour at $5 \sigma$, second contour at $15 \sigma$, then $15 \sigma$ spacing with $\sigma=$ $31 \mathrm{mJy}$ beam ${ }^{-1} \mathrm{~km} \mathrm{~s}^{-1}$ ), and ${ }^{13} \mathrm{CO} J=2 \rightarrow 1$ with $3 \sigma$ spacing contours, $\sigma=23 \mathrm{mJy}_{\text {beam }}{ }^{-1} \mathrm{~km} \mathrm{~s}^{-1}$. Dotted lines show negative contours in steps of $3 \sigma$. Bottom: ALMA observations, from left to right: ${ }^{12} \mathrm{CO} J=3 \rightarrow 2$ with uniform weighting to highlight the central gas cavity ( $3 \sigma$ spacing, $\sigma=$ $40 \mathrm{mJy}$ beam ${ }^{-1} \mathrm{~km} \mathrm{~s}^{-1}$ ), and ${ }^{13} \mathrm{CO} J=1 \rightarrow 01 \sigma$ spacing contours starting at $2 \sigma$ (for positive and negative contours), $\sigma=6.8 \mathrm{mJy} \mathrm{beam}^{-1} \mathrm{~km} \mathrm{~s}^{-1}$.

the ${ }^{12} \mathrm{CO} J=2 \rightarrow 1$ transition which extends beyond 2 ", likely as a result of a difference in opacity since the sensitivity and resolution are equivalent for these two lines. We also display the ALMA ${ }^{12} \mathrm{CO} J=3 \rightarrow 2$ emission for comparison, using uniform weighting in order to show evidence for the tiny inner gas cavity which is readily resolved in this high-resolution image. Given its very low level, the continuum emission was not subtracted in Fig. 2, meaning that this CO cavity cannot artificially result from over-subtraction of the continuum. The separation of the central emission peaks is about $0.8^{\prime \prime}$, which translates into a maximum cavity radius of about $45 \mathrm{au}$. This value is refined in the subsequent modelling presented in the following sections. The channel maps in Figs. A.1 and A.2 display the typical velocity pattern of rotating discs. In Fig. 3, the CO integrated area maps have been superimposed to the scattered light emission from the HST observations. These figures confirm that most of the molecular emission is apparently less extended than the optical dust disc. The ${ }^{13} \mathrm{CO} J=1 \rightarrow 0$ transition has a rather poor 


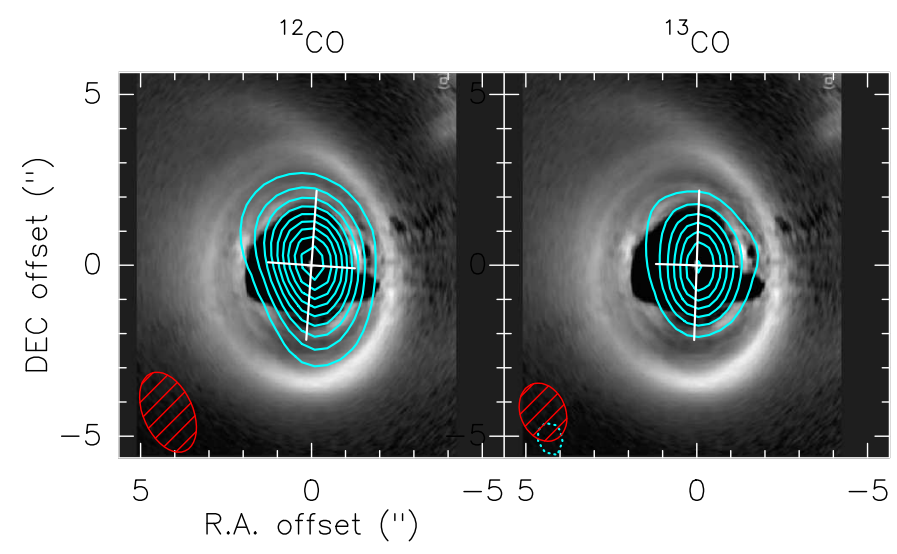

Fig. 3. Integrated intensity of the $\mathrm{CO}$ line superimposed on the HST scattered emission (Clampin et al. 2003). The cross indicates the position angle and aspect ratio as determined from gas modelling (Sect. 3). Left: ${ }^{12} \mathrm{CO} \quad J=2 \rightarrow 1$ emission, the contour spacing is $6 \sigma$, i.e. $5.5 \times 10^{-1} \mathrm{Jy} \mathrm{beam}^{-1} \mathrm{~km} \mathrm{~s}^{-1}$. Beam size: $2.48 \times 1.45^{\prime \prime}$. Right: ${ }^{13} \mathrm{CO} J=2 \rightarrow 1$ emission, with a $3 \sigma$ contour spacing, i.e. $7.8 \times 10^{-2} \mathrm{Jy} \mathrm{beam}^{-1} \mathrm{~km} \mathrm{~s}^{-1}$. Beam size: $1.76 \times 1.32^{\prime \prime}$.

sensitivity due to bad weather conditions and a large phase noise (Fig. A.3), we only use its integrated intensity in the subsequent analysis. The two transitions $\mathrm{C}^{18} \mathrm{O} J=2 \rightarrow 1(219.560 \mathrm{GHz})$ and $\mathrm{C}^{18} \mathrm{O} J=1 \rightarrow 0(109.782 \mathrm{GHz})$ are not detected in our NOEMA and ALMA projects respectively, but constraining upper limits are given in Table 3.

\subsection{Opacity of $\mathrm{CO}$ lines}

Using the line ratio of the ${ }^{12} \mathrm{CO}$ and ${ }^{13} \mathrm{CO}$ isotopologues for the same transition level, the line opacity can be estimated with the following formula:

$\mathcal{R}_{12 / 13}=\frac{S_{{ }_{12} \mathrm{CO}}}{S_{{ }^{13} \mathrm{CO}}}=\left(\frac{v_{12}}{v_{13}}\right)^{2}\left(\frac{1-e^{\tau_{12}}}{1-e^{\tau_{13}}}\right)$,

where $\tau_{12}$ and $\tau_{13}$ are the opacity of the ${ }^{12} \mathrm{CO}$ and ${ }^{13} \mathrm{CO}$ lines, respectively. If we further assume that the opacity ratio of ${ }^{12} \mathrm{CO}$ over ${ }^{13} \mathrm{CO}$ is equal to the elemental abundance ratio (Wilson \& Rood 1994), then $\tau_{12} / \tau_{13}=77$ and we can solve for Eq. (1). For the $J=2 \rightarrow 1$ transition, we measure from NOEMA observations a line ratio $\mathcal{R}_{12 / 13}=7.1 \pm 1.7$, and derive $\tau_{12}=$ $14 \pm 4$ and $\tau_{13}=0.18 \pm 0.05$. It should be noted that here we derived the $\mathrm{CO}$ opacity from the ${ }^{12} \mathrm{CO} /{ }^{13} \mathrm{CO}$ flux ratio in velocity channels of $1 \mathrm{~km} \mathrm{~s}^{-1}$ to minimise the smearing effect due to rotation (Guilloteau et al. 2006) and averaged the results. The same analysis for the $J=1 \rightarrow 0$ transition, based on our new ALMA-Band 3 measurement and the integrated intensity $S\left({ }^{13} \mathrm{CO}\right)=1.6 \pm 0.2 \mathrm{Jy} \mathrm{km} \mathrm{s}^{-1}$ reported by Flaherty et al. (2016) with CARMA yields $\tau_{12}=8 \pm 2$ and $\tau_{13}=0.11 \pm 0.02$, in good agreement with the $J=2 \rightarrow 1$ transition estimate. Even if these values could be biased by a factor of a few due to the uncertainties on the ${ }^{12} \mathrm{C} /{ }^{13} \mathrm{C}$ abundance ratio, as discussed in Kóspál et al. (2013), the ${ }^{12} \mathrm{CO}$ lines appears to be optically thick and the ${ }^{13} \mathrm{CO}$ optically thin. The ${ }^{13} \mathrm{CO}$ transition is therefore a more robust gas mass tracer than ${ }^{12} \mathrm{CO}$, and this consideration leads us to reconsider the mass of gas derived by Flaherty et al. (2016) and White et al. (2016) who assumed optically thin emission for the ${ }^{12} \mathrm{CO}$ lines. Within the uncertainties, our upper limit on $\mathrm{C}^{18} \mathrm{O}$ at $115.271 \mathrm{GHz}$ and $219.560 \mathrm{GHz}$ is consistent with ISM $\mathrm{CO}$ isotopologue abundance ratios.

\section{Modelling}

We independently model the ${ }^{12} \mathrm{CO} J=2 \rightarrow 1,{ }^{13} \mathrm{CO} J=2 \rightarrow 1$, and ${ }^{12} \mathrm{CO} J=3 \rightarrow 2$ lines with the Diskfit code (Piétu et al. 2007). We assume power laws for the CO surface density $\left(\Sigma_{\mathrm{CO}}(r)=\right.$ $\left.\Sigma_{0}(r / 100 \mathrm{au})^{-p}\right)$, the temperature $T_{\mathrm{ex}}(r)=T_{0}(r / 100 \mathrm{au})^{-q}$, and the gas velocity $V(r)=V_{0}(r / 100 \mathrm{au})^{-a_{v}}$. In this study, we fixed the velocity exponent so that $a_{\mathrm{v}}=0.5$ (Keplerian rotation). The disc is assumed to have a sharp outer edge at $R_{\text {out }}$. The vertical density is assumed to have a Gaussian profile (see Eq. (1), Piétu et al. 2007), with the scale height being a (free) power law of radius $H(r)=H_{0}(r / 100 \mathrm{au})^{-h}$. The line emission is computed assuming a (total) local line width $\mathrm{d} V$ independent of the radius and local thermodynamic equilibrium (LTE; i.e. $T_{0}$ represents the rotation temperature of the level population distribution). In addition to the above intrinsic parameters, the model also includes geometric parameters: the source position $\left(x_{0} ; y_{0}\right)$, the inclination $i$, and the position angle (PA) of the rotation axis ; and the source systemic velocity $V_{\text {sys }}$ relative to the local standard of rest (LSR) frame.

The modelling is done in the $u, v$ plane to avoid non-linear effects due to deconvolution. The minimization is completed by a Bayesian analysis using a Markov chain Monte Carlo (MCMC) code, emcee (Foreman-Mackey et al. 2013). Our approach differs from the analysis of White et al. (2016), which did not include the physical disc parameters in the modelling and focussed on the geometrical and kinematic description of the ${ }^{12} \mathrm{CO} J=3 \rightarrow 2$ emission. Our results are presented in Table 4 . Comparison with observations are shown for the integrated spectra in Fig. 4, and for the channel maps in Fig. 5.

\subsection{Spatial distribution and kinematics of the CO gas}

The spatial extent of the gas disc is constrained to consistent values around $R_{\text {out }}=275 \pm 3$ au by the two transitions of ${ }^{12} \mathrm{CO}$. This value is slightly larger (within $3 \sigma$ ) than the $224 \pm 20$ au reported by Flaherty et al. (2016) from a simultaneous fit to ${ }^{12} \mathrm{CO} J=3 \rightarrow 2$ and ${ }^{12} \mathrm{CO} J=1 \rightarrow 0$, because these latter authors fixed the density exponent to a smaller value: $p=1.5$. The ${ }^{13} \mathrm{CO}$ line emission is slightly more compact with $R_{\text {out }}=240 \pm 20 \mathrm{au}$. Although the discrepancy does not appear to be statistically significant, it may result from the combination of opacity and sensitivity effects. More interestingly, an inner cavity is confirmed with a radius $R_{\text {in }}=17 \pm 3$ au from ${ }^{12} \mathrm{CO} J=3 \rightarrow 2$, as first suggested in lowerresolution maps by Flaherty et al. (2016). The spatial resolution and sensitivity of the NOEMA observations are not sufficient to provide a robust constraint, but point towards consistent values of the inner disc edge. The ${ }^{13} \mathrm{CO} J=2 \rightarrow 1$ data suggest a larger value $R_{\text {in }}=35 \pm 5 \mathrm{au}$. This discrepancy should be taken with care because the beam size of these NOEMA data is more than four to five times larger than the ALMA beam for ${ }^{12} \mathrm{CO} J=3 \rightarrow 2$. If confirmed, this result could be explained by the difference in opacity between the two isotopologues: since the ${ }^{12} \mathrm{CO}$ transition is 70 times more optically thick than the ${ }^{13} \mathrm{CO}$ transition, a larger inner radius for ${ }^{13} \mathrm{CO}$ could be indicative of a shallow increase of the density at the inner disc edge, while a sharp edge is expected to produce similar inner radii for both lines. A similar effect has been reported in the 15-70 au gas cavity of transitional disc J160421.7-213028 (Dong et al. 2017), although the nature of the two discs is different, because HD 141569A does not show any sign of having a dust cavity (a compact continuum emission was reported at all frequencies in Sect.3.1). At the reported resolution, the presence of unresolved gaps in the gas distribution in the central 50 au may also result in a similar discrepancy 

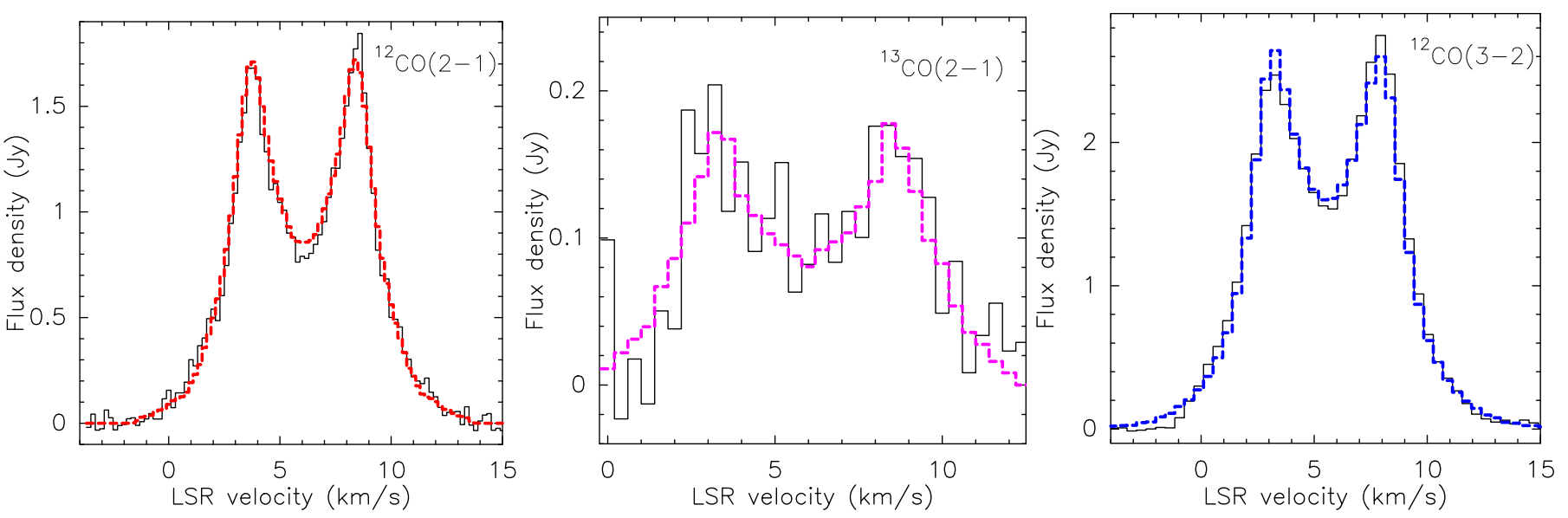

Fig. 4. Observed line profiles and superposed best models obtained with Diskfit (colour dashed lines) for, from left to right: ${ }^{12} \mathrm{CO} J=2 \rightarrow 1$, ${ }^{13} \mathrm{CO} J=2 \rightarrow 1$ NOEMA data, and ${ }^{12} \mathrm{CO} J=3 \rightarrow 2$ ALMA data.

Table 4. Results of Diskfit modelling for the ${ }^{12} \mathrm{CO}$ and ${ }^{13} \mathrm{CO}$ with $1 \sigma$ error bars.

\begin{tabular}{lccc}
\hline \hline & ${ }^{12} \mathrm{CO} J=3 \rightarrow 2$ & ${ }^{12} \mathrm{CO} J=2 \rightarrow 1$ & ${ }^{13} \mathrm{CO} J=2 \rightarrow 1$ \\
\hline$x_{0}(\mathrm{mas})$ & $-43 \pm 1$ & $-74 \pm 2$ & $-15 \pm 33$ \\
$y_{0}(\mathrm{mas})$ & $-4 \pm 1$ & $-17 \pm 4$ & $-19 \pm 28$ \\
$i\left(^{\circ}\right)$ & $56.05 \pm 0.07$ & $57.7 \pm 0.3$ & $57.2 \pm 1.4$ \\
$\mathrm{PA}\left({ }^{\circ}\right)$ & $86.60 \pm 0.06$ & $85.8 \pm 0.2$ & $88 \pm 1$ \\
$V_{\text {sys }}\left(\mathrm{km} \mathrm{s}^{-1}\right)$ & $6.05 \pm 0.01$ & $6.07 \pm 0.01$ & $5.92 \pm 0.02$ \\
$R_{\text {out }}(\mathrm{au})$ & $273 \pm 1$ & $277 \pm 3$ & $232 \pm 11$ \\
$R_{\text {in }}(\mathrm{au})$ & $17 \pm 3$ & 17 & $35 \pm 5$ \\
$a_{\mathrm{v}}$ & 0.5 & 0.5 & 0.5 \\
$V_{0}\left(\mathrm{~km} \mathrm{~s}^{-1}\right)$ & $4.44 \pm 0.01$ & $4.4 \pm 0.3$ & $4.57 \pm 0.05$ \\
$\mathrm{~d} v\left(\mathrm{~km} \mathrm{~s}{ }^{-1}\right)$ & $0.435 \pm 0.003$ & $0.41 \pm 0.01$ & $0.29 \pm 0.05$ \\
$T_{0}(\mathrm{~K})$ & $28.1 \pm 0.2$ & $33 \pm 12$ & $15_{-2}^{+3}$ \\
$q$ & $0.28 \pm 0.01$ & $0.10 \pm 0.09$ & $0.36 \pm 0.30$ \\
$\Sigma_{0}(\mathrm{CO})\left(\mathrm{cm}^{-2}\right)$ & $3.5 \pm 2.0 \times 10^{17}$ & $5.5 \pm 0.2 . \times 10^{16}$ & $4.9_{-1.1}^{+2.3} \times 10^{15}$ \\
$p$ & $2.7 \pm 0.5$ & $2.8 \pm 0.1$ & $1.8 \pm 0.5$ \\
$H_{0}(\mathrm{au})$ & $17 \pm 1$ & $17.8 \pm 0.5$ & 9 \\
$h$ & 1.25 & $1.45 \pm 0.05$ & $1.3 \pm 0.7$ \\
\hline
\end{tabular}

Notes. Parameters in italics are fixed. The physical parameters $T_{0}, \Sigma_{0}, V_{0}, H_{0}$ are scaled at a radial distance of $100 \mathrm{au}$. The offset values are given with respect to the phase centres, whose coordinates are RA = $15: 49: 57.73$, Dec $=-03: 55: 16.62$ for the ${ }^{12} \mathrm{CO} J=3 \rightarrow 2$ line, and $\mathrm{RA}=15: 49: 57.75$, Dec $=-03: 55: 16.34$ for the ${ }^{12} \mathrm{CO} J=2 \rightarrow 1$ and ${ }^{13} \mathrm{CO} J=2 \rightarrow 1$ data.

between the $\mathrm{CO}$ isotopologues. The inner rim value (17 au) for ${ }^{12} \mathrm{CO}$ is coherent with the spectroscopic detection of warm $\mathrm{CO}$ through IR ro-vibrational lines by van der Plas et al. (2015), with a modelled distribution of the gas between 14 and 45 au (after correcting for the revised stellar distance).

The inclination and position angle are found to be in the range $i=56-58^{\circ}$ and $\mathrm{PA}=86 \pm 1^{\circ}$ for ${ }^{12} \mathrm{CO}$ lines, and $i=$ $53 \pm 2^{\circ}$ and $\mathrm{PA}=90 \pm 2^{\circ}$ for ${ }^{13} \mathrm{CO}$, which is in good agreement with the optical values of $i=52.5 \pm 4.5^{\circ}$ and $\mathrm{PA}=85.4 \pm 1^{\circ}$ respectively (Augereau et al. 1999). The disc is flared, although the flaring exponent $h$ is not well constrained, a value consistent with $h=1.25$ is found for both ${ }^{12} \mathrm{CO}$ transitions, while it is naturally not constrained for the optically thin transition of ${ }^{13} \mathrm{CO}$. For ${ }^{12} \mathrm{CO}$, a scale height $H_{0}=17 \mathrm{au}$ is found at $100 \mathrm{au}$. This is twice the value of the hydrostatic scale height $\left(H_{\text {hydro }}=8 \mathrm{au}\right)$ for a disc with $T_{0}=30 \mathrm{~K}$, as measured from the optically thick transitions of ${ }^{12} \mathrm{CO}$. This is consistent with direct measurements of the ${ }^{12} \mathrm{CO}$ layer location in HD 163296 (de Gregorio-Monsalvo et al. 2013) and the Flying Saucer (Guilloteau et al. 2016), where $\mathrm{CO}$ is located at 2-3 scale heights (Dutrey et al. 2017).

In our preliminary fits, the velocity exponent proved to be very close to the Keplerian value $a_{v}=0.5$, and we adopted this fixed value for the MCMC exploration reported in Table 4. At $100 \mathrm{au}$, the rotation velocity is better constrained for ${ }^{12} \mathrm{CO}$ lines, with a value $V_{0}=4.44 \pm 0.01 \mathrm{~km} \mathrm{~s}^{-1}$ that can be converted into a central stellar mass of $2.22 \pm 0.01 M_{\odot}$. This value is slightly smaller than the mass of $2.39 \pm 0.05 M_{\odot}$ reported by White et al. (2016), but is very consistent with the spectral analysis of Merín et al. (2004) that yielded $M_{\star}=2.00_{-0.15}^{+0.18} M_{\odot}$. The ${ }^{13} \mathrm{CO}$ analysis leads to a slightly larger mass $M_{\star}=2.35 \pm 0.05 M_{\odot}$.

\subsection{Temperature and density laws}

The modelling of the two optically thick transitions ${ }^{12} \mathrm{CO} J=2 \rightarrow 1$ and ${ }^{12} \mathrm{CO} J=3 \rightarrow 2$ provides very consistent estimates for the excitation temperature, $T_{0} \sim 30 \mathrm{~K}$ with a relatively flat radial profile $q<0.4(q=0.28 \pm 0.01$ for ${ }^{12} \mathrm{CO} J=3 \rightarrow 2$ and $q=0.10 \pm 0.09$ for $\left.{ }^{12} \mathrm{CO} J=2 \rightarrow 1\right)$. The temperature is ill constrained for the optically thin ${ }^{13} \mathrm{CO}$ transition. A value of $30 \mathrm{~K}$ is consistent with the analysis of the ${ }^{12} \mathrm{CO} J=3 \rightarrow 2$ detection with SMA by Flaherty et al. (2016, who used a fixed temperature exponent $q=0.5$ and found $T_{0}=33_{-4}^{+11} \mathrm{~K}$ ). It is somewhat smaller than the gas temperature of about $50 \mathrm{~K}$ derived from ${ }^{12} \mathrm{CO}$ line for a similar, albeit younger and more optically thick disc around the Herbig Ae star MWC 480 (Piétu et al. 2007). Given the star luminosity of $30 L_{\odot}$, blackbody grains in an optically thin disc are predicted to have a temperature of $60 \mathrm{~K}$ at a distance of $100 \mathrm{au}$, and would be much hotter than the detected gas. The derived gas temperature is also significantly smaller than the range of kinetic temperatures computed by Thi et al. (2014) with their Prodimo modelling based on unresolved detections of [OI], $\mathrm{C}[\mathrm{II}]$ and ${ }^{12} \mathrm{CO} J=3 \rightarrow 2$ lines around HD 141569. In their modelling, the gas is heated by Polycyclic Aromatic Hydrocarbons (PAH) and cooled by [OI] and $\mathrm{CO}$ lines, and the computed temperature reaches about $100 \mathrm{~K}$ at $100 \mathrm{au}$ in the inner disc and drops down to $40 \mathrm{~K}$ at $500 \mathrm{au}$ (i.e. well beyond the outer edge of the emission we detect with ALMA/NOEMA). The excitation temperature we 

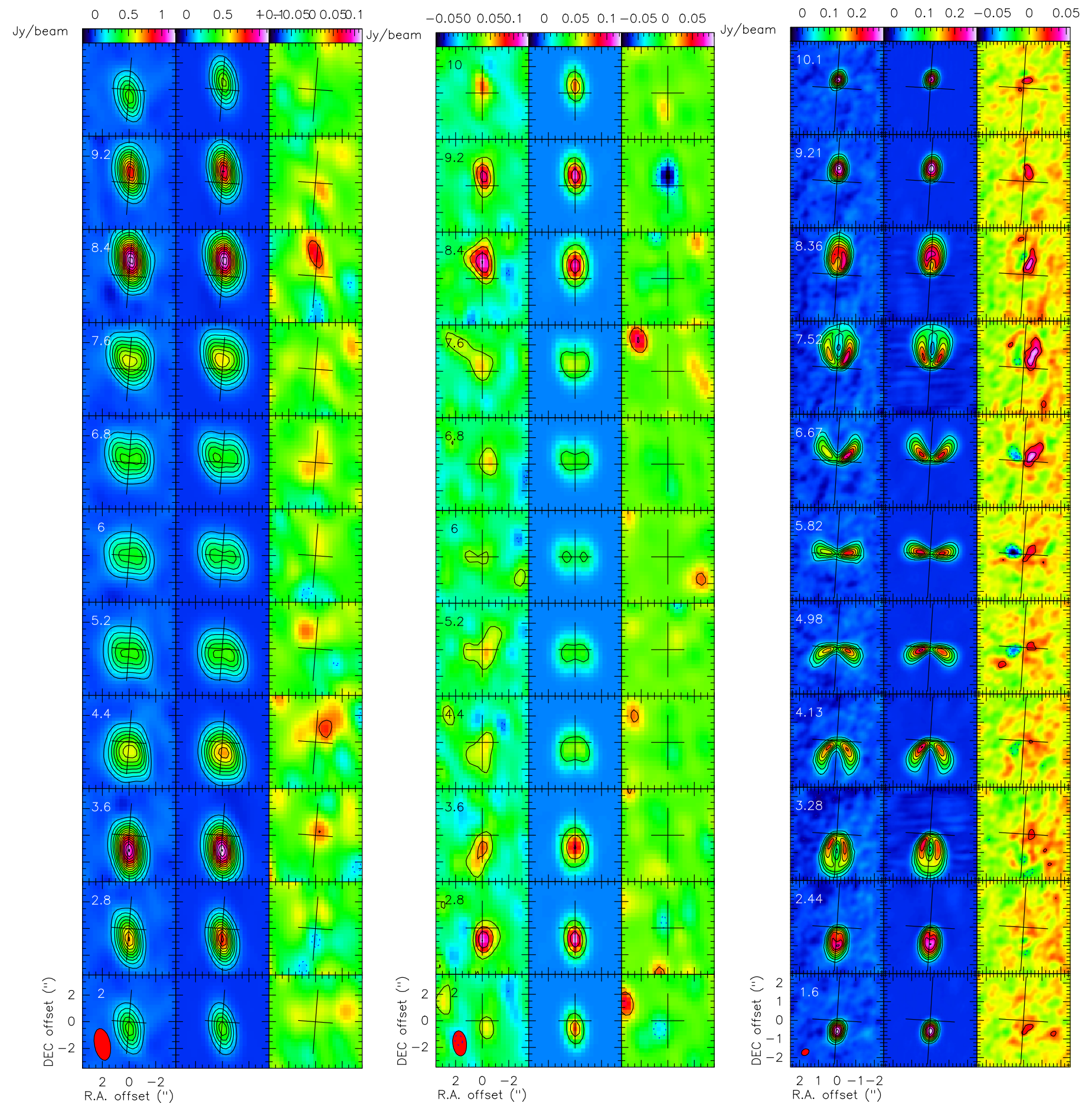

Fig. 5. Results of the modelling showing the channel maps for the transition (from left to right) ${ }^{12} \mathrm{CO} J=2 \rightarrow 1,{ }^{13} \mathrm{CO} J=2 \rightarrow 1$ (NOEMA) and ${ }^{12} \mathrm{CO} J=3 \rightarrow 2$ (ALMA). In each sub-panel, we present from left to right: the observations, the model (see best-fit parameters in Table 4), and the residual emission. The latter is obtained by subtraction in the $u, v$ plane before imaging and cleaning. Contour spacing is $5 \sigma$ for the ${ }^{12} \mathrm{CO}$ transitions $\left(\sigma=16 \mathrm{mJy}_{\text {beam }}{ }^{-1}\right.$ for $J=2 \rightarrow 1$ and $\sigma=6.3 \mathrm{mJy}_{\text {beam }}{ }^{-1}$ for $\left.J=3 \rightarrow 2\right)$, and $3 \sigma$ for the ${ }^{13} \mathrm{CO} J=2 \rightarrow 1$ transition $\left(\sigma=10 \mathrm{mJy}^{2}\right.$ beam $\left.{ }^{-1}\right)$. Residual contour spacing is $3 \sigma$ for all transitions.

derive from ${ }^{12} \mathrm{CO}$ may still be smaller than the actual gas kinetic temperature. Low values of the gas temperature (although with a much more dramatic deviation) have also been reported in the hybrid disc HD 21997 by Kóspál et al. (2013), where the excitation temperature derived from $\mathrm{CO}$ line ratios drops to values as low as $6-9 \mathrm{~K}$. Flaherty et al. (2016) propose that this low gas temperature could be explained by enhanced cooling from CO (following Hollenbach \& Tielens 1999), and/or NLTE effects (following Matrà et al. 2015).

In the absence of $\mathrm{C}^{18} \mathrm{O}$ emission, we use the more optically thin emission of the ${ }^{13} \mathrm{CO} J=2 \rightarrow 1$ line to constrain the $\mathrm{CO}$ surface density. Our modelling yields a best-fit value $\Sigma_{0}=$ $5_{-1}^{+2} \times 10^{15} \mathrm{~cm}^{-2}$ at 100 au with an exponent $p=1.8 \pm 0.5$. We note that the two other transitions of ${ }^{12} \mathrm{CO}$ lead to much steeper 


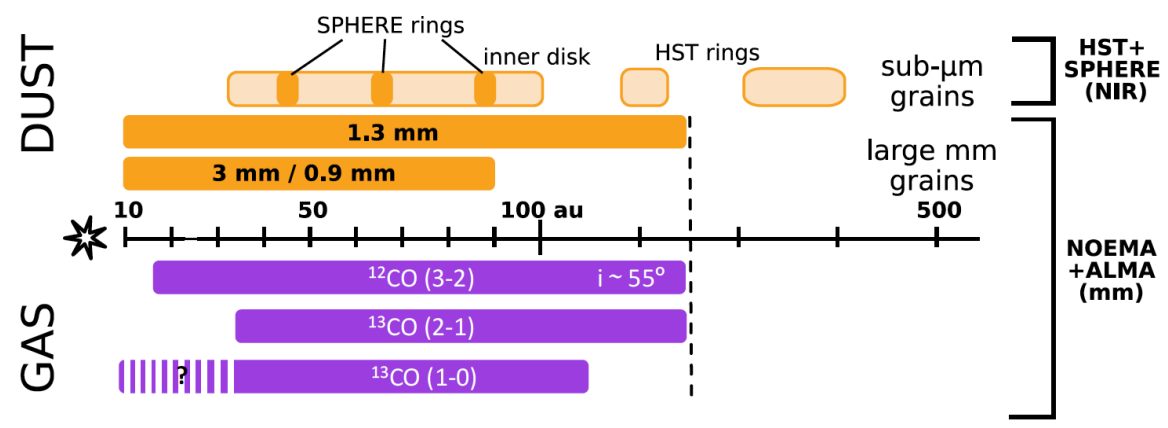

Fig. 6. Global scheme of dust and gas distribution in HD 141569 summarizing the most recent resolved observations. profiles with $p \sim 3$ and require a surface density of the order of $6 \times 10^{16} \mathrm{~cm}^{-2}$. These values of the surface density are at least one order of magnitude smaller than those found for the younger, gas-rich disc around the Herbig Ae star MWC 480: Piétu et al. (2007) report, at a distance of $100 \mathrm{au}, \Sigma_{0}=8 \times 10^{16} \mathrm{~cm}^{-2}$ for ${ }^{13} \mathrm{CO}$ and $6 \times 10^{18} \mathrm{~cm}^{-2}$ for ${ }^{12} \mathrm{CO}$. This system is taken here as a reference as it is one of the very few where the surface density of ${ }^{13} \mathrm{CO}$ has been precisely constrained through similar disc emission modelling.

We derived a total mass of CO gas based on $\Sigma_{0}$ and used the minimum outer disc edge value $R_{\text {out }}=232$ au inferred from the ${ }^{13} \mathrm{CO}$ line (hence a minimum mass value). Because most of the mass lies in the inner regions with a power law distribution, the computed value is sensitive to the inner disc edge, and we take into account the inner cavity detected with the ${ }^{12} \mathrm{CO}$ emission in the ALMA high-resolution maps $\left(R_{\text {in }}=\right.$ $17 \mathrm{au})$. Assuming a standard isotopic ratio ${ }^{12} \mathrm{CO} /{ }^{13} \mathrm{CO}=77$, these parameters yield $M_{{ }^{12} \mathrm{CO}}=0.10_{-0.02}^{+0.05} M_{\oplus}\left(=4 \times 10^{23} \mathrm{~kg}\right)$, where we take into account the uncertainties on $R_{\text {in }}, R_{\text {out }}, \Sigma_{0}$ and $p$. This value is 50 times larger than the $\mathrm{CO}$ mass reported by White et al. (2016) based on the ${ }^{12} \mathrm{CO}$ emission.

The conversion to a total mass of gas is very uncertain. A low CO content compared to the dust emission was already noted in the early detection of CO isotopologues in DM Tau (Dutrey et al. 1997). Several recent studies (Bergin et al. 2013; Reboussin et al. 2015) favour much lower values for the $\left[\mathrm{CO} / \mathrm{H}_{2}\right]$ ratio than the canonical ISM value of $10^{-4}$ (see also the discussion on carbon depletion in e.g. Miotello et al. 2017). We discuss this point again in Sect. 5.2. Here, in order to compare with other works, we nevertheless convert this minimum mass of $\mathrm{CO}$ into $M_{\mathrm{H}_{2}}=71_{-14}^{+36} M_{\oplus}$ or $0.21_{-0.04}^{+0.11} M_{\mathrm{Jup}} \simeq 2.10^{-4} M_{\odot}$ using the standard ISM abundance. Using the grid of models developed by Miotello et al. (2016), which include the effect of isotope selective photodissociation, the luminosity of the ${ }^{13} \mathrm{CO} J=2 \rightarrow 1$ line and the non-detection of $\mathrm{C}^{18} \mathrm{O} J=2 \rightarrow 1$ favours a total mass in the range $1-5 \times 10^{-4} M_{\odot}$, in good agreement with the previous estimate. This is one order of magnitude larger than the value reported by Flaherty et al. (2016) from the SMA detection of ${ }^{12} \mathrm{CO} J=3 \rightarrow 2$, but is in good agreement with the earlier modelling of Thi et al. (2014) and Jonkheid et al. (2006), based on spatially unresolved observations of the same line, who found a total gas mass in the range $67-164 M_{\oplus}$. Our value is a factor of three to five smaller than the total gas mass of $6-9 \times 10^{-4} M_{\odot}$ reported by Miley et al. (2018) based on the integrated flux of the optically thin transition ${ }^{13} \mathrm{CO} J=2 \rightarrow 1$, but these remain in reasonable agreement due to the different methodologies used. This total gas mass value is clearly on the lower bound of the range of disc masses found for Herbig Ae stars, and usually derived from mm dust flux converted to gas mass with an assumed gas-todust ratio of 100 (see, e.g. Williams \& Cieza 2011). It is instead typical of discs around very low-mass TTS (for instance, TTS disc masses obtained from ${ }^{13} \mathrm{CO}$ and $\mathrm{C}^{18} \mathrm{O} J=3 \rightarrow 2$ in Lupus range between $10^{-5}$ and $10^{-3} M_{\odot}$, based on models assuming isotope selective photo-dissociation and $\mathrm{CO}$ freeze-out on grains Miotello et al. 2017). HD 141569 also harbours one of the most massive molecular gas components among the gas-rich debris discs reported in Moór et al. (2017) based on supposedly optically thin CO isotopologues. Even if we underestimate the $\mathrm{CO}$ mass due to ${ }^{13} \mathrm{CO}$ optical depth effects or the uncertain $\left[\mathrm{CO} / \mathrm{H}_{2}\right]$ abundance, such a low mass supports the process of ongoing gas dissipation in this 5 Myr-old disc.

\section{Discussion}

\subsection{Comparison with optical/NIR emission}

The distribution of matter is strikingly different at optical/NIR and $\mathrm{mm}$ wavelengths. A global sketch summarizing and comparing the respective radial extent of the main disc components detected at optical/NIR and mm wavelengths is presented in Fig. 6. The continuum emission at $\mathrm{mm}$ wavelength is clearly much more compact than the $250-400$ au broad rings of the debris disc revealed at optical/NIR wavelengths, and is also more compact and centrally peaked than the gas emission characterised in the following section. Furthermore, it is coincident with the innermost component recently reported by various authors in the NIR domain. Indeed, in the inner $100 \mathrm{au}$ recent observations with VLT/SPHERE have revealed the presence of a series of concentric rings and arcs with semi-major axes in the range 45-90 au (Perrot et al. 2016). The link between the $\mu \mathrm{m}$ grains responsible for the IR emission and the $\mathrm{mm}$ grains detected with ALMA/NOEMA cannot be clarified because of the limited resolution of our data. VLT/VISIR observations in the mid-IR also suggest the presence of an even more internal component (Thi et al. 2014), which was recently detected at $L^{\prime}$ band with the Keck vortex coronagraph within $70 \mathrm{au}$ (Mawet et al. 2017) and may extend up to $100 \mathrm{au}$. According to the extended scattered-light emission reported with HST/STIS by Konishi et al. (2016), this inner warm component may also be spatially associated with the inner rim of the gas disc that our modelling locates around $15-20$ au from ${ }^{12} \mathrm{CO} J=3 \rightarrow 2$ maps (similarly to the warm CO detected in spectroscopy in the 14-60 au region by Goto et al. 2006; van der Plas et al. 2015). The disc is not classified as a transitional disc because of the lack of a mm dust cavity. A small cavity of no more than 15 au in radius is observed in the $\mathrm{CO}$ maps, consistent with the earlier spectroscopic detections of a warm CO ring, but the continuum emission is strikingly centrally peaked. Inside this gas cavity, there is probably not much material: VLTI-PIONIER observations in the $H$-band reported a completely unresolved structure (Lazareff et al. 2017), which might be dominated by the stellar emission itself (accounting for $86 \%$ of the total flux), without suggestion of an inner ring near the sublimation distance of small grains. 

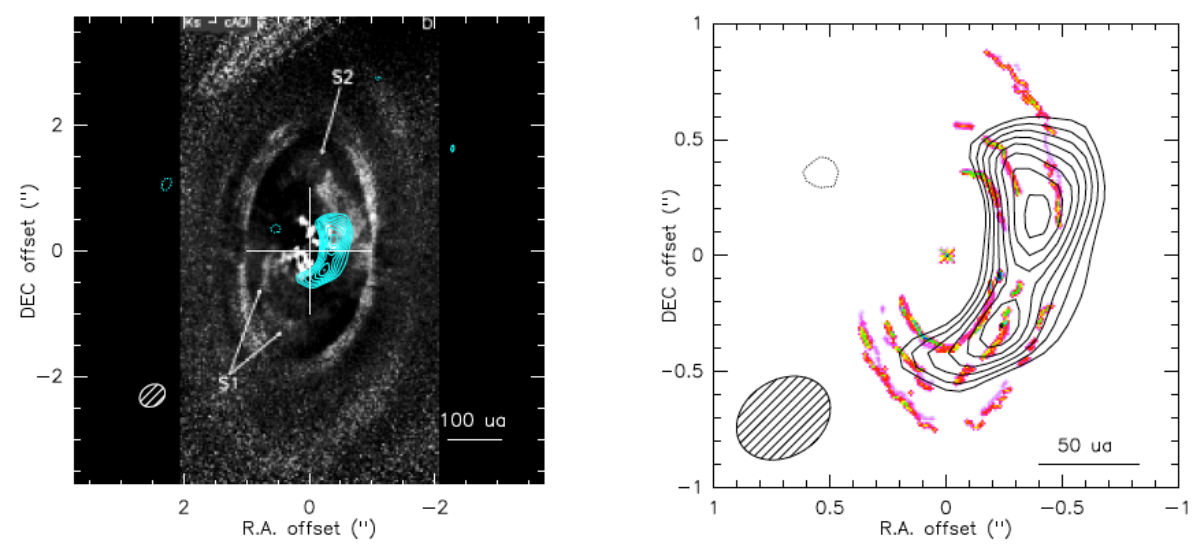

Fig. 7. Montage of the ${ }^{12} \mathrm{CO} J=3 \rightarrow 2$ residual emission with the series of concentric rings detected in NIR scattered light with SPHERE by Perrot et al. (2016).
The scattered light images show many asymmetries at various spatial scales, with spirals, arcs, and multiple ring structures. The outer limit of the gas disc is precisely coincident with the so-called "inner ring" near 250 au reported previously. Among the possible ring formation mechanisms, Takeuchi \& Artymowicz (2001) proposed a complex mechanism based on the outward migration of small grains at the outer edge of a gas disc. HD 141569 is the only resolved disc so far that displays this combination of distant dust rings and a gas-rich inner disc.

Large-scale asymmetries are also suggested in both wavelength domains. In Fig. 7, we superimpose the SPHERE/ $K \mathrm{~s}$ image on the map of our best-fit residual of ${ }^{12} \mathrm{CO} J=$ $3 \rightarrow 2$ ALMA emission. This residual emission, which was already reported by White et al. (2016), is significant (up to $6 \sigma)$ at all velocity channels and peaks between 6 and $9 \mathrm{~km} \mathrm{~s}^{-1}$ (see Fig. 5). We present here the integrated emission, which extends on the western side with an elliptical shape. It displays two brightness peaks centred at $60-70$ au of deprojected distance at azimuth angles of about $220^{\circ}$ and $290^{\circ}$, in the region of the three concentric rings detected with SPHERE. Since the ${ }^{12} \mathrm{CO}$ emission is optically thick and the feature is not detected in the isotopologues due to insufficient sensitivity and angular resolution, we cannot disentangle between a local temperature offset and a density feature. However, the significance of this ${ }^{12} \mathrm{CO}$ brightness increase, the coincidence with the location of the inner rings, and the similar brightness asymmetry raise questions about the interplay between gas and dust in this region. Lyra \& Kuchner (2013) proposed a mechanism based on the development of a clumping instability to create eccentric rings from the coupling of gas and grains, but this appears to be efficient in a regime of low gas-to-dust ratio, which does not seem to be favoured in the inner region (see discussion in Sect. 5.2). The $250 \mathrm{au}$ dust ring (and possibly also the outermost $400 \mathrm{au}$ ring) at the edge of the detectable gaseous component (see Fig. 3) may instead be an ideal location to reach the necessary very low value of gas-to-dust ratio $(G / D<1)$ for such an instability to develop. So far, no planetary companion candidate has been reported in the inner 100 au region with a sensitivity limit of the order of 1-3 $M_{\text {Jup }}$ (Perrot et al. 2016). The destruction of planetesimals (by collision or evaporation) could produce both large quantities of small grains and release substantial amounts of gas, as has been suggested by the detection of an asymmetrical emission of CO in $\beta$ Pictoris (Dent et al. 2014). In HD 141569, such a collisional event or enhanced planetesimal evaporation might be more difficult to demonstrate because of the large residual amount of primordial gas in the region of the NIR rings.

\subsection{Gas-to-dust ratio}

The global gas-to-dust mass ratio in the disc is expected to trace the evolution of the system, but getting an accurate value is challenging because of the numerous assumptions that must be made especially in the computation of the total gas mass, as discussed in the previous section. The mass of dust is no less uncertain as it relies on the unknown value of the dust mass opacity $\kappa_{v}$, and we will follow the prescription: $\kappa_{v}=0.02(\lambda / 1 \mathrm{~mm})^{-\beta} \mathrm{cm}^{2} \mathrm{~g}^{-1}$, which is similar to that adopted by Beckwith et al. (1990) (with $\beta=1) . M_{\text {dust }}$ can be estimated on the basis of the mm dust flux $\left(F_{v}\right)$, which is constrained in our observations at three different frequencies. From the modelling of the SED, earlier models by Jonkheid et al. (2006) and Thi et al. (2014) showed that the dust emission is optically thin from optical to $\mathrm{mm}$ wavelengths, meaning that the mm flux can be used to provide a robust estimate of the dust mass. Using the mm dust fluxes reported in Table 1 , the extreme values of $\beta$ in the range [0,0.7], and typical values of the temperature for dust grains located at 50-100 au from this $30 L_{\odot}$ star $\left(T_{\mathrm{d}}=65-90 \mathrm{~K}\right), M_{\text {dust }}$ is computed with the standard equation:

$M_{d}=\frac{F_{v} D^{2} c^{2}}{2 h v^{3} \kappa_{v}}\left(\exp \left(\frac{h v}{k_{\mathrm{B}} T_{\mathrm{d}}}\right)-1\right)$.

We obtain values for $M_{\text {dust }}$ in the range $0.03-0.52 M_{\oplus}$. This value is significantly smaller than for other Herbig Ae systems. In order to compare with dust masses derived for other gas-rich debris discs reported in Moór et al. (2017), we alternatively used the same dust opacity $\left(\kappa_{v=230 \mathrm{GHz}}=2.3 \mathrm{~cm}^{2} \mathrm{~g}^{-1}\right)$ and their assumed temperature $\left(T_{\mathrm{d}}=50 \mathrm{~K}\right)$, which leads to $M_{d}=0.46 M_{\oplus}$, which makes it the most massive gas-rich debris disc with HD 131488 in this study. With our gas mass estimate, $70 M_{\oplus}$, and our first choice of dust opacity, yielding $M_{\text {dust }} \sim 0.03-0.52 M_{\oplus}$, we can obtain a gas-to-dust mass ratio $G / D$ in the range 135-2370. This is most likely a severe lower limit, since we assumed a high $\mathrm{CO} / \mathrm{H}_{2}$ abundance ratio to derive the gas mass. In T Tauri discs, it is in general necessary to invoke an overall depletion of $\mathrm{CO}$ by a factor of ten or so to bring the derived G/D ratio to the ISM value of 100 (see, Piétu et al. 2007; Williams \& Best 2014), although the warmer discs around HAeBe stars have higher $\mathrm{CO}$ /dust ratios, and can sometimes be directly interpreted by $G / D=100$ and a normal $\mathrm{CO} / \mathrm{H}_{2}$ ratio (e.g. AB Aur Piétu et al. 2007, or also HD 163296 in Williams \& Best 2014). The estimated gas-to-dust mass ratio appears at least one order of magnitude larger than the values reported in Miotello et al. (2017) for a sample of sources in Lupus, where gas mass estimates were also derived from optically thin $\mathrm{CO}$ isotopologues 
(although with more complex models including isotope selective processes, as mentioned earlier).

This analysis must furthermore be refined because the morphology of the dust and gas components is quite different. The $G / D$ ratio can obviously display ample radial variations due to migration of large grains towards the inner disc regions, or local enhancements of the gas and dust density. The resolved images of the HD 141569 disc suggest that the bulk of the mm grains reside in a small disc confined within $R_{\text {out,dust }} \sim 100 \mathrm{au}$, while the gas reservoir extends between about 20 and $270 \mathrm{au}$. There is possible evidence for a second, low-emission component of dust grains in the lower-resolution NOEMA maps (and concurrent hints at very short ALMA baselines), which could extend out to the limit of the gas disc and may contribute up to $30 \%$ of the dust emission. We checked however that the spatial variations of $G / D$ do not affect the overall conclusion. Similar large values of the $G / D$ ratio have also been reported in the $30 \mathrm{Myr}$ old hybrid disc HD 21997 by Kóspál et al. (2013): the authors quote a CO-to-dust ratio of $0.4-0.9$ within $R_{\text {out }}=150$ au that converts to 280-630 for $\mathrm{H}_{2}$-to-dust. However, the morphology of the two systems is very different, with a dust cavity within 55 au for HD 21997, but no evidence for a gas cavity. We also reported in Péricaud et al. (2017) the tendency of gas-rich debris discs to display a larger ratio of $\mathrm{CO}$ to $\mathrm{mm}$ continuum flux compared to other Herbig/TTauri systems, which could suggest that there is a larger gas-to-dust ratio in this transient phase. Beyond 270 au, the abrupt rarefaction of the gas naturally decreases $G / D$, although neither $\mathrm{mm}$ grains nor gas emission are detected in this cold region. The outermost rings can be regarded as a nascent debris disc, where the grains detected in scattered light emission are produced by a collisional cascade from a reservoir of unseen parent bodies.

\subsection{Origin of the gas}

From the optically thin ${ }^{13} \mathrm{CO}$ emission, we derived a ${ }^{12} \mathrm{CO}$ gas mass in the range $8-15 \times 10^{-2} M_{\oplus}$, a factor of 50 larger than the estimate by White et al. (2016) based on the optically thick ${ }^{12} \mathrm{CO}$ transition, and a factor of 2 larger than the gas mass derived by Miley et al. (2018) based on optically thin ${ }^{13} \mathrm{CO}$ observations. The gas disc appears also twice more massive than the $\mathrm{C}^{18} \mathrm{O}$ mass estimate in the 30 Myr-old hybrid disc HD 21997 (Kóspál et al. 2013). Based on the typical lifetime for self-shielded CO molecules and using estimates of $\mathrm{CO}$ content and gas production in solar system comets, these latter authors computed that more than 6000 Hale-Bopp-like comets would have to be destroyed each year to reproduce the observed level of CO in HD 21997 if the gas were of secondary origin. In the hypothesis of an exocometary origin, $\mathrm{CO}$ molecules can only be protected through self-shielding, which is however a more efficient mechanism for ${ }^{12} \mathrm{CO}$ than for its isotopologues. We estimate the lifetime of self-shielded ${ }^{13} \mathrm{CO}$ molecules from Visser et al. (2009) using the gas column density derived from our previous analysis and adopting $T_{\mathrm{ex}}=50 \mathrm{~K}$, and we find $860 \mathrm{yr}$ as a maximum value under the ambient interstellar UV field (the actual lifetime might even be shorter considering the additional contribution of the stellar UV field). Using the mass measured for the optically thin molecule ${ }^{13} \mathrm{CO}$ and assuming a standard isotopic ratio, the ${ }^{12} \mathrm{CO}$ production rate required to sustain the observed amount of ${ }^{13} \mathrm{CO}$ is $4.7 \times 10^{20} \mathrm{~kg} \mathrm{yr}^{-1}$, and the ${ }^{12} \mathrm{CO}$ production rate is $3 \times 10^{21} \mathrm{~kg} \mathrm{yr}^{-1}$, a value more than 2000 times larger than the gas production rate inferred for the $\beta$ Pic disc by Dent et al. (2014) and Matrà et al. (2017). In Péricaud et al. (2017), we alternatively calculated the maximum amount of ${ }^{12} \mathrm{CO}$ that can be locked in comets and planetesimals for a massive Herbig Ae $\operatorname{disc}\left(M_{\mathrm{d}}=0.1 M_{\star}\right)$, assuming that $10 \%$ of the total amount of available oxygen in the disc can ultimately be evaporated in the form of $\mathrm{CO}$ (an upper limit derived from solar system comets). We found an upper limit for the initial ${ }^{12} \mathrm{CO}$ mass in comets and planetesimals of about $4 \times 10^{25} \mathrm{~kg}$, hence a ${ }^{13} \mathrm{CO}$ mass of $5.2 \times 10^{23} \mathrm{~kg}$, which is 100 times the $\mathrm{CO}$ mass value estimated for HD 141569. With a ${ }^{13} \mathrm{CO}$ lifetime of about $860 \mathrm{yr}$, the observed mass of ${ }^{13} \mathrm{CO}$ would imply a replenishment rate of $6 \times 10^{18} \mathrm{~kg} \mathrm{yr}^{-1}$, and hence a maximum lifetime of the initial ${ }^{13} \mathrm{CO}$ reservoir of less than $10^{5} \mathrm{yr}$. This value is likely an upper limit because we overestimated the $\mathrm{CO}$ content in comets relying on CO-rich comets only and assumed a very massive disc. It is however much shorter than the system age of $5 \pm 3$ Myr (Merín et al. 2004).

Moreover, the morphology of the $\mathrm{CO}$ emission, which continuously extends from 15-20 au out to 275 au favours a primordial origin for the gas, in contrast to the localised emission detected around $\beta$ Pic where the gas and dust are likely produced in a planetesimal belt (Matrà et al. 2017). Our modelling of the CO maps shows that the gas disc is well represented by a protoplanetary-like disc with a surface density an order of magnitude lower than in the typical 1-2 Myr-old discs around Herbig Ae stars. The total amount of dust and gas in this system is similar to the older debris discs around HD 21997, HD 121617, HD 131488 and HD 131835 (Moór et al. 2017). No large inner cavity is found in HD 141569 in contrast to HD 21997 or HD 121617, indicating possible different mechanisms at play. A possible alternative scenario was recently proposed by Kral et al. (2017, 2019), in which even the most massive hybrid discs could be explained by secondary $\mathrm{CO}$ gas shielded by accumulated neutral carbon that is a photodissociation product of molecular gas released by planetesimals. This would require specific (low) values of the viscosity to accumulate a sufficient amount of $\mathrm{C}^{0}$, and it is uncertain whether this scenario could efficiently be applied to such a young system as HD 141569A.

The detection of other molecular species in the gas phase can provide a complementary diagnosis on the nature of the gas and reveal the specific chemistry in such a system. Our attempt to find $\mathrm{HCO}^{+}, \mathrm{H}_{2} \mathrm{CO}, \mathrm{HCN}, \mathrm{HC}_{3} \mathrm{~N}$ and $\mathrm{CS}$ with the IRAM-30 m radio antenna did not provide any successful detection (see Sect. 2.3). With our sensitivity limit, we would have been able to detect $\mathrm{HCO}^{+} J=1 \rightarrow 0$ and CS $J=3 \rightarrow 2$ lines at $>20 \sigma$ if these were as bright as in the disc of AB Aur (Pacheco-Vázquez et al. 2015). However, the comparison should account for the smaller size of the HD 141569 disc, which is 250 au in radius, instead of about $500 \mathrm{au}$ for that of AB Aur, and of the differences in distance. We therefore conservatively conclude that the average brightness for these transitions is at least a factor of three below that found for $\mathrm{AB}$ Aur. This may be the result of lower column densities or lower excitation temperatures. Both possibilities favour a low gas surface density in HD 141569. The presence of a prominent OI line (in addition to CII) revealed by Herschel also underlines the similarity with other Herbig Ae discs, because this feature is not detected in any of the more evolved, gas-rich debris discs (Meeus et al. 2012; Moór et al. 2016). The presence of prominent PAH features is another argument favouring the presence of primordial material in at least some regions of the disc, since such strong features have not been detected in IR spectra of Vega-like stars so far (e.g. Chen et al. 2006). This again reinforces its classification as an evolved disc around a Herbig Ae star rather than a debris disc. The disc is thus likely dominated by primordial gas in the phase of dissipation, and differs from its less evolved siblings due to its reduced CO surface density, and most notably 
its extremely low continuum which suggests a faster evolution of the dust component as already highlighted in Péricaud et al. (2017).

Acknowledgements. This work was supported by "Programme National de Physique Stellaire" (PNPS from INSU/CNRS.) This research made use of the SIMBAD database, operated at the CDS, Strasbourg, France. This paper makes use of the following ALMA data: ADS/JAO.ALMA2012.1.00698.S and ADS/JAO.ALMA.2013.1.00883.S. ALMA is a partnership of ESO (representing its member states), NSF (USA), and NINS (Japan), together with NRC (Canada), NSC and ASIAA (Taiwan), and KASI (Republic of Korea) in cooperation with the Republic of Chile. The Joint ALMA Observatory is operated by ESO, AUI/NRAO, and NAOJ. This paper is also based on observations carried out with the IRAM NOEMA interferometer and the IRAM 30-m telescope. IRAM is supported by INSU/CNRS (France), MPG (Germany), and IGN (Spain). We thank the anonymous referee for his/her careful reading and useful suggestions to improve the manuscript.

\section{References}

Andrews, S. M., Wilner, D. J., Espaillat, C., et al. 2011, ApJ, 732, 42 Andrews, S. M., Huang, J., Pérez, L. M., et al. 2018, ApJ, 869, L41

Augereau, J. C., Lagrange, A. M., Mouillet, D., \& Ménard, F. 1999, A\&A, 350, L51

Avenhaus, H., Quanz, S. P., Garufi, A., et al. 2018, ApJ, 863, 44

Beckwith, S. V. W., Sargent, A. I., Chini, R. S., \& Guesten, R. 1990, AJ, 99, 924

Bergin, E. A., Cleeves, L. I., Gorti, U., et al. 2013, Nature, 493, 644

Boccaletti, A., Augereau, J.-C., Marchis, F., \& Hahn, J. 2003, ApJ, 585, 494

Chen, C. H., Sargent, B. A., Bohac, C., et al. 2006, ApJS, 166, 351

Clampin, M., Krist, J. E., Ardila, D. R., et al. 2003, AJ, 126, 385

Currie, T., Grady, C. A., Cloutier, R., et al. 2016, ApJ, 819, L26

de Gregorio-Monsalvo, I., Ménard, F., Dent, W., et al. 2013, A\&A, 557, A133

Dent, W. R. F., Wyatt, M. C., Roberge, A., et al. 2014, Science, 343, 1490

Dong, R., van der Marel, N., Hashimoto, J., et al. 2017, ApJ, 836, 201

Dutrey, A., Guilloteau, S., \& Guelin, M. 1997, A\&A, 317, L55

Dutrey, A., Lecavelier Des Etangs, A., \& Augereau, J. C. 2004, Comets II, eds M. C. Festou, H. U. Keller, \& H. A. Weaver (Tucson, AZ: University of Arizona Press), 81

Dutrey, A., Guilloteau, S., Piétu, V., et al. 2017, A\&A, 607, A30

Espaillat, C., Muzerolle, J., Najita, J., et al. 2014, Protostars and Planets VI (Tucson, AZ: University of Arizona Press), 497

Fedele, D., van den Ancker, M. E., Henning, T., Jayawardhana, R., \& Oliveira, J. M. 2010, A\&A, 510, A72

Flaherty, K. M., Hughes, A. M., Andrews, S. M., et al. 2016, ApJ, 818, 97

Foreman-Mackey, D., Hogg, D. W., Lang, D., \& Goodman, J. 2013, PASP, 125 306

Gaia Collaboration (Brown, A. G. A., et al.) 2018, A\&A, 616, A1

Garufi, A., Benisty, M., Pinilla, P., et al. 2018, A\&A, 620, A94

Goto, M., Usuda, T., Dullemond, C. P., et al. 2006, ApJ, 652, 758

Guilloteau, S., Piétu, V., Dutrey, A., \& Guélin, M. 2006, A\&A, 448, L5

Guilloteau, S., Dutrey, A., Piétu, V., \& Boehler, Y. 2011, A\&A, 529, A105

Guilloteau, S., Piétu, V., Chapillon, E., et al. 2016, A\&A, 586, L1

Haisch, K. E. J., Lada, E. A., \& Lada, C. J. 2001, ApJ, 553, L153

Hollenbach, D. J., \& Tielens, A. G. G. M. 1999, Rev. Mod. Phys., 71, 173

Hughes, A. M., Duchêne, G., \& Matthews, B. C. 2018, ARA\&A, 56, 541

Jonkheid, B., Kamp, I., Augereau, J.-C., \& van Dishoeck, E. F. 2006, A\&A, 453, 163
Konishi, M., Grady, C. A., Schneider, G., et al. 2016, ApJ, 818, L23

Kóspál, Á., Moór, A., Juhász, A., et al. 2013, ApJ, 776, 77

Kral, Q., Matrà, L., Wyatt, M. C., \& Kennedy, G. M. 2017, MNRAS, 469, 521

Kral, Q., Marino, S., Wyatt, M. C., Kama, M., \& Matra, L. 2019, Turk. J. Phys., 43,126

Lazareff, B., Berger, J.-P., Kluska, J., et al. 2017, A\&A, 599, A85

Lieman-Sifry, J., Hughes, A. M., Carpenter, J. M., et al. 2016, ApJ, 828, 25

Lyra, W., \& Kuchner, M. 2013, Nature, 499, 184

Matrà, L., Panić, O., Wyatt, M. C., \& Dent, W. R. F. 2015, MNRAS, 447, 3936

Matrà, L., Dent, W. R. F., Wyatt, M. C., et al. 2017, MNRAS, 464, 1415

Mawet, D., Choquet, É., Absil, O., et al. 2017, AJ, 153, 44

Meeus, G., Montesinos, B., Mendigutía, I., et al. 2012, A\&A, 544, A78

Merín, B., Montesinos, B., Eiroa, C., et al. 2004, A\&A, 419, 301

Miley, J. M., Panić, O., Wyatt, M., \& Kennedy, G. M. 2018, A\&A, 615, L10

Miotello, A., van Dishoeck, E. F., Kama, M., \& Bruderer, S. 2016, A\&A, 594 A85

Miotello, A., van Dishoeck, E. F., Williams, J. P., et al. 2017, A\&A, 599, A113

Moór, A., Ábrahám, P., Juhász, A., et al. 2011, ApJ, 740, L7

Moór, A., Kóspál, Á., Ábrahám, P., et al. 2016, ApJ, 826, 123

Moór, A., Curé, M., Kóspál, Á., et al. 2017, ApJ, 849, 123

Mouillet, D., Lagrange, A. M., Augereau, J. C., \& Ménard, F. 2001, A\&A, 372, L61

Nilsson, R., Liseau, R., Brandeker, A., et al. 2010, A\&A, 518, A40

Pacheco-Vázquez, S., Fuente, A., Agúndez, M., et al. 2015, A\&A, 578, A81

Pérez, L. M., Carpenter, J. M., Chandler, C. J., et al. 2012, ApJ, 760, L17

Péricaud, J., Di Folco, E., Dutrey, A., Guilloteau, S., \& Piétu, V. 2017, A\&A, 600, A62

Perrot, C., Boccaletti, A., Pantin, E., et al. 2016, A\&A, 590, L7

Piétu, V., Dutrey, A., \& Guilloteau, S. 2007, A\&A, 467, 163

Pinilla, P., Birnstiel, T., Ricci, L., et al. 2012, A\&A, 538, A114

Reboussin, L., Guilloteau, S., Simon, M., et al. 2015, A\&A, 578, A31

Reche, R., Beust, H., \& Augereau, J.-C. 2009, A\&A, 493, 661

Schreyer, K., Guilloteau, S., Semenov, D., et al. 2008, A\&A, 491, 821

Sylvester, R. J., Skinner, C. J., Barlow, M. J., \& Mannings, V. 1996, MNRAS, 279, 915

Sylvester, R. J., Dunkin, S. K., \& Barlow, M. J. 2001, MNRAS, 327, 133

Takeuchi, T., \& Artymowicz, P. 2001, ApJ, 557, 990

Thi, W.-F., Pinte, C., Pantin, E., et al. 2014, A\&A, 561, A50

van der Marel, N., van Dishoeck, E. F., Bruderer, S., et al. 2016, A\&A, 585, A58

van der Marel, N., Williams, J. P., Ansdell, M., et al. 2018, ApJ, 854, 177

van der Plas, G., van den Ancker, M. E., Waters, L. B. F. M., \& Dominik, C. 2015, A\&A, 574, A75

Visser, R., van Dishoeck, E. F., \& Black, J. H. 2009, A\&A, 503, 323

Weinberger, A. J., Becklin, E. E., Schneider, G., et al. 1999, ApJ, 525, L53

Weinberger, A. J., Rich, R. M., Becklin, E. E., Zuckerman, B., \& Matthews, K. 2000, ApJ, 544, 937

White, J. A., \& Boley, A. C. 2018, ApJ, 859, 103

White, J. A., Boley, A. C., Hughes, A. M., et al. 2016, ApJ, 829, 6

White, J. A., Boley, A. C., MacGregor, M. A., Hughes, A. M., \& Wilner, D. J. 2018, MNRAS, 474, 4500

Williams, J. P., \& Best, W. M. J. 2014, ApJ, 788, 59

Williams, J. P., \& Cieza, L. A. 2011, ARA\&A, 49, 67

Wilson, T. L., \& Rood, R. 1994, ARA\&A, 32, 191

Wyatt, M. C. 2008, ARA\&A, 46, 339

Wyatt, M. C., Panić, O., Kennedy, G. M., \& Matrà, L. 2015, Ap\&SS, 357, 103

Zhu, Z., Nelson, R. P., Hartmann, L., Espaillat, C., \& Calvet, N. 2011, ApJ, 729, 47

Zuckerman, B., Forveille, T., \& Kastner, J. H. 1995, Nature, 373, 494 


\section{Appendix A: Channel maps for the ${ }^{12} \mathrm{CO}$ and ${ }^{13} \mathrm{CO}$ lines}

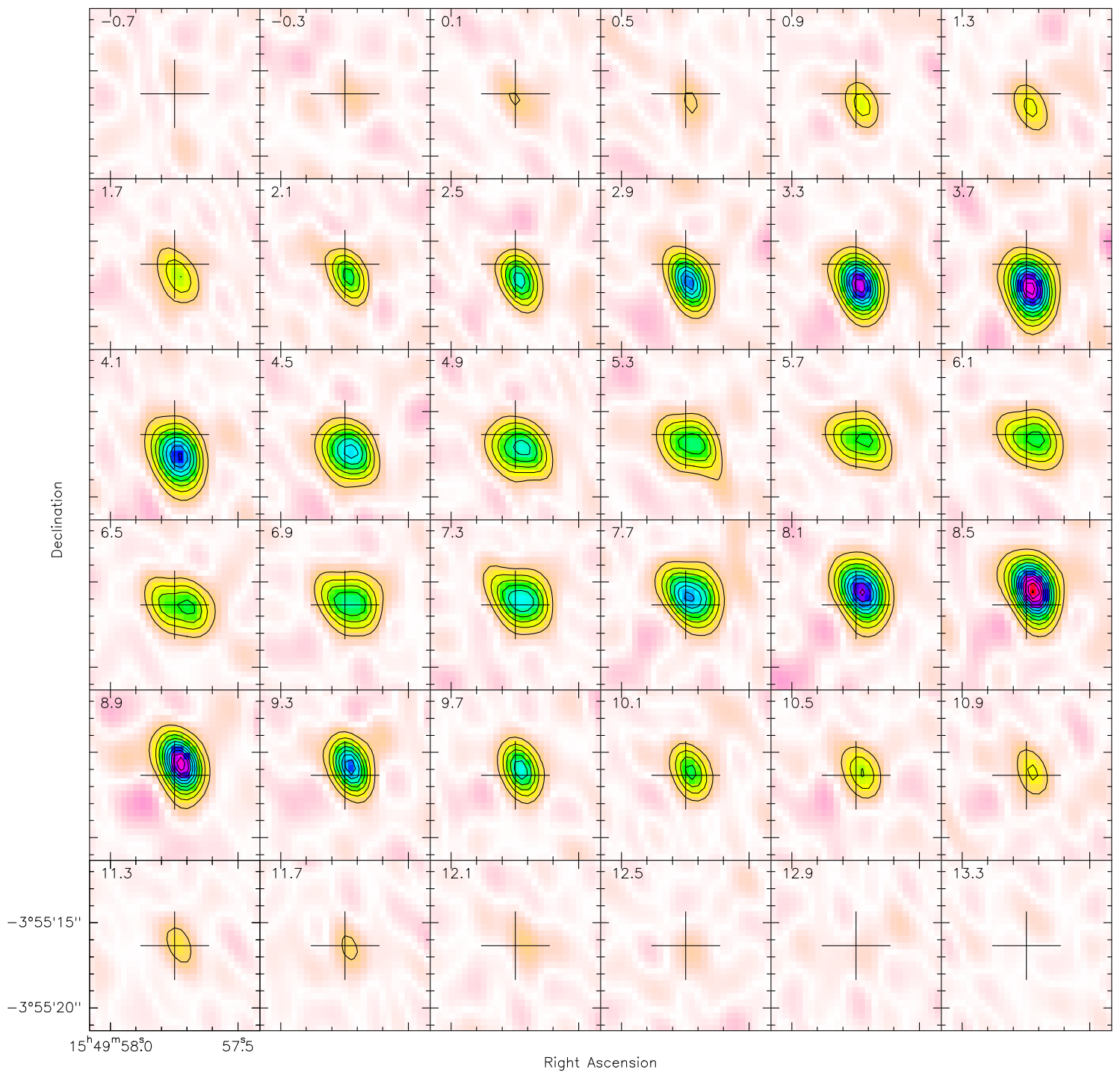

Fig. A.1. Channel map for the ${ }^{12} \mathrm{CO} J=2 \rightarrow 1$ transition observed with NOEMA, the spatial resolution is $2.5 \times 1.4^{\prime \prime}$, the contour spacing is $7 \sigma$. 
E. Di Folco et al.: An ALMA/NOEMA study of HD 141569A hybrid disc

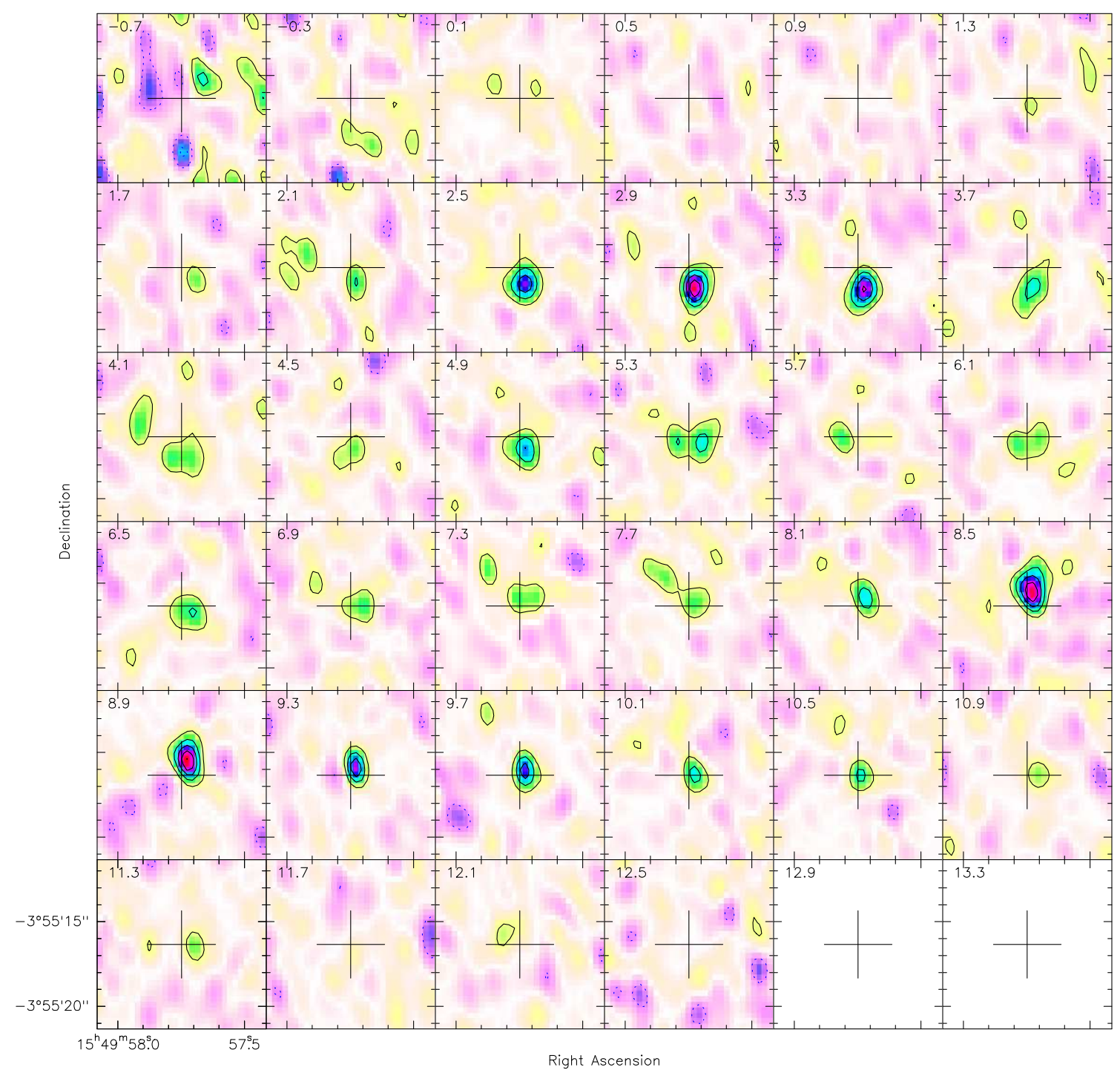

Fig. A.2. Channel map for the ${ }^{13} \mathrm{CO} J=2 \rightarrow 1$ transition observed with NOEMA, the spatial resolution is $1.9 \times 1.1^{\prime \prime}$, the contour spacing is $2 \sigma$, same channel selection as for Fig. A.1. 


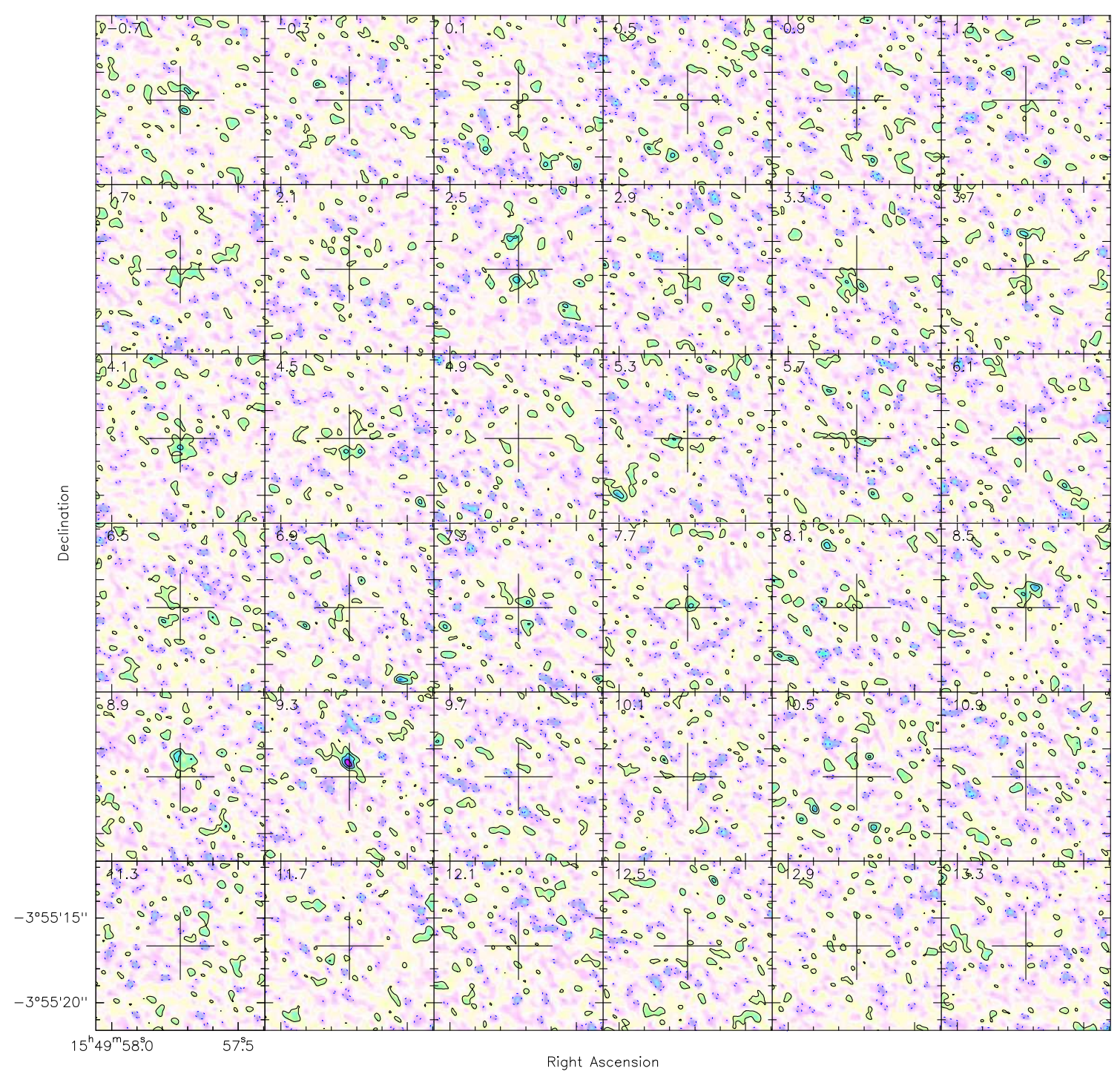

Fig. A.3. Channel map for the ${ }^{13} \mathrm{CO} J=1 \rightarrow 0$ transition observed with ALMA, the spatial resolution is $0.7 \times 0.5^{\prime \prime}$, the contour spacing is $3 \sigma$, same channel selection as for Fig. A.1. 